\title{
Quantum Algorithm for Time-Dependent Hamiltonian Simulation by Permutation Expansion
}

\author{
Yi-Hsiang Chen $\odot,{ }^{1,3, *}$ Amir Kalev, ${ }^{2}$ and Itay Hen ${ }^{1,3}$ \\ ${ }^{1}$ Information Sciences Institute, University of Southern California, Marina del Rey, California 90292, USA \\ ${ }^{2}$ Information Sciences Institute, University of Southern California, Arlington, Virginia 22203, USA \\ ${ }^{3}$ Department of Physics and Astronomy, and Center for Quantum Information Science \& Technology, University \\ of Southern California, Los Angeles, California 90089, USA
}

(Received 21 April 2021; accepted 25 August 2021; published 9 September 2021)

\begin{abstract}
We present a quantum algorithm for the dynamical simulation of time-dependent Hamiltonians. Our method involves expanding the interaction-picture Hamiltonian as a sum of generalized permutations, which leads to an integral-free Dyson series of the time-evolution operator. Under this representation, we perform a quantum simulation for the time-evolution operator by means of the linear combination of unitaries technique. We optimize the time steps of the evolution based on the Hamiltonian's dynamical characteristics, leading to a gate count that scales with an $L^{1}$-norm-like scaling with respect only to the norm of the interaction Hamiltonian, rather than that of the total Hamiltonian. We demonstrate that the cost of the algorithm is independent of the Hamiltonian's frequencies, implying its advantage for systems with highly oscillating components, and for time-decaying systems the cost does not scale with the total evolution time asymptotically. In addition, our algorithm retains the near optimal $\log (1 / \epsilon) / \log \log (1 / \epsilon)$ scaling with simulation error $\epsilon$.
\end{abstract}

DOI: 10.1103/PRXQuantum.2.030342

\section{INTRODUCTION}

The problem of simulating quantum systems, whether it is to study their dynamics, or to infer their salient equilibrium properties, was the original motivation for quantum computers [1] and remains one of their major potential applications [2,3]. Classical algorithms for this problem are known to be grossly inefficient. Nonetheless, a significant fraction of the world's computing power today is spent on solving instances of this problem - a reflection on their importance [4-6].

An important class of quantum simulations that is known to be particularly challenging, and is the focus of this work, is that of time-dependent quantum processes, which are at the heart of many important quantum phenomena. These include, for example, quantum control schemes [7], transition states of chemical reactions [8], analog quantum computers such as quantum annealers [9], and the quantum approximate optimization algorithm [10]. Devising state-of-the-art resource efficient quantum algorithms

*yihsianc@usc.edu

Published by the American Physical Society under the terms of the Creative Commons Attribution 4.0 International license. Further distribution of this work must maintain attribution to the author(s) and the published article's title, journal citation, and DOI. to simulate these types of processes on quantum circuits is therefore a very worthy cause: it will allow for the studying of said phenomena in a controllable and vastly more illuminating manner.

In the literature, a number of quantum algorithms designed to simulate the dynamics of time-dependent quantum many-body Hamiltonians already exist. However, most of them are variants of algorithms that suit time-independent Hamiltonians but lack optimizations for dynamical ones. For example, Hamiltonians based on the Lie-Trotter-Suzuki decomposition were developed in Refs. $[11,12]$, where the complexity scales polynomially with error. More recent advances $[13,14]$ improve it to a logarithmic error scaling, which directly lead to applications in time-dependent Hamiltonian simulations [15,16]. A recent study by Berry et al. [17] improves the Hamiltonian scaling to $L^{1}$-norm, by considering the dynamical properties of the time-dependent Hamiltonian. However, these mostly comprise of slicing the dynamics into a sequence of "quasistatic" steps, each of which implementing a static quantum simulation module. In addition, all the abovementioned algorithms assume a time-dependent oracle - a straightforward but not necessarily practical assumption that can obscure the true complexity of the simulation when physical models are considered.

The suboptimality that characterizes existing quantum algorithms can be attributed mainly to the fact that the 
time-evolution operator for time-dependent Hamiltonians is a more intricate entity than its time-independent counterpart (this matter is discussed in more detail below). While in the time-independent case the Schrödinger equation can be formally integrated, the time-evolution unitary operator for time-dependent systems is given in terms of a Dyson series [18] - a perturbative expansion, wherein each summand is a multidimensional integral over a time-ordered product of the (usually interaction-picture) Hamiltonian at different points in time. These time-ordered integrals pose multiple algorithmic and implementation challenges.

In this paper, we provide a quantum algorithm for simulating a time-dependent Hamiltonian dynamics. This algorithm invokes a separation of the Hamiltonian $H(t)$ into a sum of a static diagonal part $H_{0}$ and a dynamical part $V(t)$, i.e., $H(t)=H_{0}+V(t)$, and switches to the interaction picture with respect to $H_{0}$. The target evolution operator becomes a product of an interaction-picture unitary $U_{I}(t)$ followed by a diagonal unitary $e^{-i H_{0} t}$ that can be simulated efficiently. The interaction Hamiltonian $V(t)$ is expanded as a sum of generalized permutations, and the resulting Dyson series of the evolution operator $U_{I}(t)$ becomes an integral-free representation [19] with the notion of divided differences, which is a well-studied quantity [20-25]. The divided differences have an intuition of discretized derivatives and is closely related to polynomial interpolations [20]. We refer the reader to Appendix A for a short summary of the notion of the divided differences. Under this representation, we use the linear combination of unitaries (LCU) method [14] to simulate $U_{I}(t)$ with a truncated Dyson series. We find a partitioning scheme that determines the duration of the time steps along the simulation. Following this procedure, in general, each time interval has a different duration that is determined from the Hamiltonian's dynamical characteristics and can lead to a substantially fewer number of steps as compared to using identical-length simulation segments, typically used in quantum simulation algorithms. We analyze the implementation gate and qubit costs and discuss the circumstances under which our simulation algorithm provides improvements over the state of the art. Specifically, our algorithm is independent of the oscillation frequencies of the Hamiltonian. This is in stark contrast to existing algorithms that have dependence on $\|d H(t) / d t\|$, which grows with oscillation rates. Another class of Hamiltonians for which our algorithm is preferred over others is those with exponential decays in time. We show that, for these systems, our algorithms require asymptotically a finite number of steps that does not scale with the evolution time, leading in turn to an exponential saving comparing to the linear scaling in existing approaches. Moreover, the cost with Hamiltonian norm only mainly depends on the interaction Hamiltonian $V(t)$ and not the total Hamiltonian $H(t)$ [17]. This also indicates an advantage of the algorithm when the time-dependent Hamiltonian is dominant by a static part.
The paper is organized as follows. In Sec. II, we review the permutation expansion method that leads to an integralfree representation for the Dyson series, as introduced in Ref. [19]. In Sec. III, we present in detail the simulation algorithm that combines the integral-free expression of the evolution operator with the LCU method, and analyze the circuit costs. We highlight the main advantages of our algorithm in Sec. III D 3. In Sec. III E, we address the cases when the exponential-sum expansion of the time dependence is not exact and estimate the error that stems from a finite sum approximation. Finally, we give a brief summary for our methods and results in Sec. V.

\section{PERMUTATION EXPANSION METHOD FOR TIME-DEPENDENT HAMILTONIANS}

In this section, we briefly describe the integral-free Dyson series expression of the evolution operator, derived from a permutation expansion of the time-dependent Hamiltonian [19]. Without loss of generality [26], we expand a general time-dependent Hamiltonian in terms of products of time-dependent diagonal matrices, $D_{i}(t)$, and permutation operators, $P_{i}$, i.e.,

$$
H(t)=\sum_{i=0}^{M} D_{i}(t) P_{i},
$$

where $P_{0} \equiv \mathbb{1}$. This decomposition can be done efficiently as long as $M$ scales polynomially with $\log d$, where $d$ is the dimension of the Hamiltonian. We decompose each diagonal matrix into a finite sum of exponential functions, i.e.,

$$
D_{i}(t)=\sum_{k=1}^{K_{i}} \exp \left(\Lambda_{i}^{(k)} t\right) D_{i}^{(k)},
$$

where $\Lambda_{i}^{(k)}$ and $D_{i}^{(k)}$ are complex diagonal matrices with diagonal elements being

$$
\begin{aligned}
& \lambda_{i, z}^{(k)} \equiv\left\langle z\left|\Lambda_{i}^{(k)}\right| z\right\rangle, \\
& d_{i, z}^{(k)} \equiv\left\langle z\left|D_{i}^{(k)}\right| z\right\rangle,
\end{aligned}
$$

in some basis $\{|z\rangle\}$ (the basis in which $D_{0}$ is diagonal) and $K_{i}$ indicates the number of terms in the exponential decomposition for $D_{i}(t)$. This can be done for many cases when the time dependencies are simple combinations of exponential terms. For simplicity, we assume here that the $K_{i}$ are finite, and address the most general time dependence in detail in Sec. III E and refer to various algorithms [27-31] for efficiently finding an exponential sum approximation of a function. 
For a lighter notation, we set $K_{i}=K$ for all $i$. We can evaluate the time-evolution operator $U(t)$ corresponding to $H(t)$ as

$$
\begin{aligned}
U(t) \equiv & \mathcal{T} \exp \left[-i \int_{0}^{t} H\left(t^{\prime}\right) d t^{\prime}\right] \\
= & \sum_{q=0}^{\infty}(-i)^{q} \int_{0}^{t} d \tau_{q} \cdots \int_{0}^{\tau_{2}} d \tau_{1} H\left(\tau_{q}\right) \cdots H\left(\tau_{1}\right) \\
= & \sum_{q=0}^{\infty} \sum_{\mathbf{i}_{q}} \sum_{\mathbf{k}_{q}}(-i)^{q} \int_{0}^{t} d \tau_{q} \cdots \\
& \times \int_{0}^{\tau_{2}} d \tau_{1} \exp \left(\Lambda_{i_{q}}^{\left(k_{q}\right)} \tau_{q}\right) D_{i_{q}}^{\left(k_{q}\right)} P_{i_{q}} \cdots \\
& \times \exp \left(\Lambda_{i_{1}}^{\left(k_{1}\right)} \tau_{1}\right) D_{i_{1}}^{\left(k_{1}\right)} P_{i_{1}},
\end{aligned}
$$

where $\mathbf{i}_{q}=\left\{i_{q}, \ldots, i_{1}\right\}$ and $\mathbf{k}_{q}=\left\{k_{q}, \ldots, k_{1}\right\}$ are multiindices. The action of $U(t)$ on a basis vector $|z\rangle$ is

$$
\begin{aligned}
& U(t)|z\rangle=\sum_{q=0}^{\infty} \sum_{\mathbf{i}_{q}} \sum_{\mathbf{k}_{q}}(-i)^{q} \int_{0}^{t} d \tau_{q} \cdots \int_{0}^{\tau_{2}} d \tau_{1} \\
& \times \exp \left(\lambda_{i_{q}, \mathbf{z}_{q}}^{\left(k_{q}\right)} \tau_{q}+\cdots \lambda_{i_{1}, \mathbf{i}_{1}}^{\left(k_{1}\right)} \tau_{1}\right) d_{i_{q}, \mathbf{z}_{q}}^{\left(k_{\mathbf{i}}\right)} \cdots d_{i_{1}, \mathbf{z}_{1}}^{\left(k_{1}\right)} \\
& \times P_{i_{q}} \cdots P_{i_{1}}|z\rangle \\
& =\sum_{q=0}^{\infty} \sum_{\mathbf{i}_{q}} \sum_{\mathbf{k}_{q}}(-i)^{q} \int_{0}^{t} d \tau_{q} \cdots \int_{0}^{\tau_{2}} d \tau_{1} \\
& \times \exp \left(\lambda_{i_{q}, \mathbf{z}_{q}}^{\left(k_{q}\right)} \tau_{q}+\cdots+\lambda_{i_{1}, \mathbf{i}_{1}}^{\left(k_{1}\right)} \tau_{1}\right) d_{\mathbf{i}_{q}, z}^{\left(\mathbf{k}_{q}\right)} P_{\mathbf{i}_{q}}|z\rangle,
\end{aligned}
$$

where $\left|z_{\mathbf{i}_{j}}\right\rangle \equiv P_{i_{j}} \cdots P_{i_{1}}|z\rangle$ with $j$ ranging from 1 to $q$, and $\lambda_{i_{j}, z_{\mathbf{i}_{j}}}^{\left(k_{j}\right)}\left(d_{i_{j}, \mathbf{i}_{\mathbf{i}_{j}}}^{\left(k_{j}\right)}\right)$ is the $z_{\mathbf{i}_{j}}$ th diagonal element of $\Lambda_{i_{j}}^{\left(k_{j}\right)}\left(D_{i_{j}}^{\left(k_{j}\right)}\right)$. We use $P_{\mathbf{i}_{q}}$ as shorthand for $P_{i_{q}} \cdots P_{i_{1}}$, and similarly $d_{\mathbf{i}_{q}, z}^{\left(\mathbf{k}_{q}\right)} \equiv d_{i_{q}, \mathbf{i}_{q}}^{\left(k_{\mathbf{i}_{q}}\right.} \cdots d_{i_{1}, \mathbf{z}_{1}}^{\left(k_{1}\right)}$. Figure 1 illustrates the accumulative actions of $D_{i}^{(k)} P_{i}$ on a basis vector $|z\rangle$.

To proceed, we use the following identity to simplify the expression in terms of divided differences. It is a variant of Hermite-Genocchi formula [20] applying to the exponential function.

Identity 1. For $\lambda_{1}, \ldots, \lambda_{q} \in \mathbb{C}$,

$$
\int_{0}^{1} d s_{q} \cdots \int_{0}^{s_{2}} d s_{1} e^{\left(\lambda_{1} s_{1}+\cdots+\lambda_{q} s_{q}\right)}=e^{\left[x_{1}, \ldots, x_{q}, 0\right]}
$$

where $x_{j}=\sum_{l=j}^{q} \lambda_{l}$ and $e^{\left[x_{1}, \ldots, x_{q}, 0\right]}$ is the divided difference of the exponential function with inputs $x_{1}, \ldots, x_{q}, 0$. (The case with $q=1$ can be shown by explicit integration, and the identity follows by induction. For more details, see Ref. [19].)
With this property, the multidimensional integration in the time-evolution operator can be simplified as

$$
\begin{aligned}
U(t)|z\rangle= & \sum_{q=0}^{\infty} \sum_{\mathbf{i}_{q}} \sum_{\mathbf{k}_{q}}(-i)^{q} \int_{0}^{t} d \tau_{q} \cdots \int_{0}^{\tau_{2}} d \tau_{1} \\
& \times \exp \left(\lambda_{i_{q}, \mathbf{z}_{q}}^{\left(k_{q}\right)} \tau_{q}+\cdots+\lambda_{i_{1}, \mathbf{i}_{1}}^{\left(k_{1}\right)} \tau_{1}\right) d_{\mathbf{i}_{q}, z}^{\left(\mathbf{k}_{q}\right)} P_{\mathbf{i}_{q}}|z\rangle \\
= & \sum_{q=0}^{\infty} \sum_{\mathbf{i}_{q}} \sum_{\mathbf{k}_{q}}(-i t)^{q} \int_{0}^{1} d s_{q} \cdots \int_{0}^{s_{2}} d s_{1} \\
& \times \exp \left[t\left(\lambda_{i_{q}, \mathbf{i}_{i}}^{\left(k_{q}\right)} s_{q}+\cdots+\lambda_{i_{1}, \mathbf{z}_{1}}^{\left(k_{1}\right)} s_{1}\right)\right] d_{\mathbf{i}_{q}, z}^{\left(\mathbf{k}_{q}\right)} P_{\mathbf{i}_{q}}|z\rangle \\
= & \sum_{q=0}^{\infty} \sum_{\mathbf{i}_{q}} \sum_{\mathbf{k}_{q}}(-i)^{q} e^{t\left[x_{1}, x_{2}, \ldots, x_{q}, 0\right]} d_{\mathbf{i}_{q}, z}^{\left(\mathbf{k}_{q}\right)} P_{\mathbf{i}_{q}}|z\rangle,
\end{aligned}
$$

where $x_{j}=\sum_{l=j}^{q} \lambda_{i_{l}, z_{i_{1}}}^{\left(k_{l}\right)}$. The second equality uses the change of variable $d \tau=t d s$, and the last equality follows from Identity 1 and the identity of $t^{q} e^{\left[t x_{0}, \ldots, t x_{q}\right]}=e^{t\left[x_{0}, \ldots, x_{q}\right]}$. By completing the basis, we get

$$
\begin{aligned}
U(t) & =\sum_{z} U(t)|z\rangle\langle z| \\
& =\sum_{z} \sum_{q=0}^{\infty} \sum_{\mathbf{i}_{q}} \sum_{\mathbf{k}_{q}}(-i)^{q} e^{t\left[x_{1}, x_{2}, \ldots, x_{q}, 0\right]} d_{\mathbf{i}_{q}, z}^{\left(\mathbf{k}_{q}\right)} P_{\mathbf{i}_{q}}|z\rangle\langle z| .
\end{aligned}
$$

This is an integral-free expression for the unitary timeevolution operator of the time-dependent Hamiltonian $H(t)$. We will later approximate the unitary by truncating the series at some order $q=Q$ that scales as $\mathcal{O}[\log (1 / \epsilon) / \log \log (1 / \epsilon)]$ [14], where $\epsilon$ is the required accuracy.

\section{TIME-DEPENDENT HAMILTONIAN SIMULATION ALGORITHM}

A time-dependent Hamiltonian $H(t)$ can be expressed as a sum of two Hamiltonians - a time-independent $H_{0}$ and a dynamical $V(t)$, i.e.,

$$
H(t)=H_{0}+V(t)
$$

In many practical models, $H_{0}$ represents a static and simple Hamiltonian that is often diagonal in a known basis (which we will identify with the computational basis). Hence, hereafter, we assume that $H_{0}$ is a diagonal operator with real diagonal elements. The $V(t)$ component represents the nontrivial interactions between subsystems. Assume [32] that $H_{0}$ is diagonal in the computational basis $\{|z\rangle\}$. We 


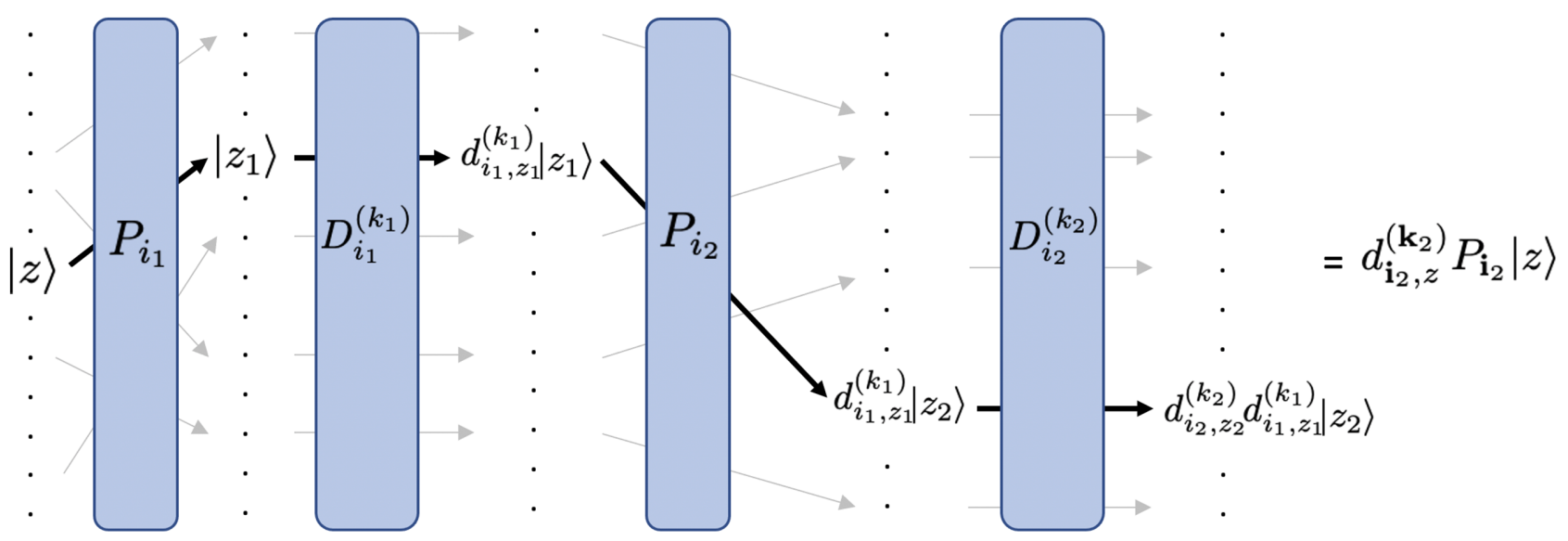

FIG. 1. The actions of a sequence of generalized permutations. This figure gives a pictorial illustration on how the elements of the diagonal matrices are picked up when interleaving with permutations. In this example, we have $q=2$.

switch to the interaction picture, i.e.,

$$
\frac{d}{d t}|\psi(t)\rangle=-i H(t)|\psi(t)\rangle \rightarrow \frac{d}{d t}\left|\psi_{I}(t)\right\rangle=-i H_{I}(t)\left|\psi_{I}(t)\right\rangle
$$

where

$$
\left|\psi_{I}(t)\right\rangle=e^{i H_{0} t}|\psi(t)\rangle \quad \text { and } \quad H_{I}(t)=e^{i H_{0} t} V(t) e^{-i H_{0} t} .
$$

The Schrödinger-picture unitary operator $U(t)$, satisfying $|\psi(t)\rangle=U(t)|\psi(0)\rangle$, is equivalent to a time-ordered matrix exponential followed by a diagonal unitary, i.e.,

$$
\begin{aligned}
U(t) & =e^{-i H_{0} t} \mathcal{T} \exp \left[-i \int_{0}^{t} H_{I}\left(t^{\prime}\right) d t^{\prime}\right] \\
& =e^{-i H_{0} t} \mathcal{T} \exp \left[-i \int_{0}^{t} e^{i H_{0} t^{\prime}} V\left(t^{\prime}\right) e^{-i H_{0} t^{\prime}} d t^{\prime}\right] .
\end{aligned}
$$

Hence, the simulation of $U(t)=e^{-i H_{0} t} U_{I}(t)$ consists of two parts - a complicated $U_{I}(t)$ and a simple diagonal unitary $e^{-i H_{0} t}$. The simulation of $e^{-i H_{0} t}$ can be achieved with a gate cost that scales only linearly with the locality of $H_{0}$ (the highest weight of terms in $H_{0}$ ). When we write $H_{0}=\sum_{\gamma=0}^{L} J_{\gamma} Z_{\gamma}$, where each $Z_{\gamma}$ is some tensor product of (single-qubit) Pauli- $Z$ operators acting on at most $d$ qubits (locality $d$ ), it can be shown that the gate cost scales as $\mathcal{O}(L d)[33,34]$. Therefore, the main focus of our simulation is on $U_{I}$.

We next provide an overview of the simulation algorithm in Sec. III A. In Sec. III B, we incorporate the LCU framework with the permutation expansion method. Section III C 2 provides the state preparation operation and Sec. III D evaluates the simulation cost for the whole procedure.

\section{A. An overview of the algorithm}

Our proposed simulation algorithm consists of a permutation expansion procedure for $U_{I}$ and the LCU method for the quantum simulation. In Sec. III B, we explain in detail the essential ingredients for merging these two approaches. Before delving into technical details, we provide an overview of the algorithm in this section.

Given a time-dependent Hamiltonian $H(t)$, we first decompose $H(t)$ into a sum of a static diagonal term $H_{0}$ (if it exists) and a dynamical term $V(t)$. We switch to an interaction picture so that the target unitary evolution $U(T)$ over a period $T$ becomes

$$
\begin{aligned}
U(T) & =e^{-i H_{0} T} \mathcal{T} \exp \left[-i \int_{0}^{T} e^{i H_{0} t} V(t) e^{-i H_{0} t} d t\right] \\
& \equiv e^{-i H_{0} T} U_{I}(T) .
\end{aligned}
$$

Therefore, the simulation of $U(T)$ is equivalent to applying $U_{I}(T)$ followed by $e^{-i H_{0} T}$. Since the diagonal unitary $e^{-i H_{0} T}$ can be efficiently simulated, we focus on $U_{I}(T)$ hereafter.

Let us expand $V(t)$ as a sum of permutations as

$$
V(t)=\sum_{i=0}^{M} D_{i}(t) P_{i},
$$

where the $P_{i}$ are permutations $\left(P_{0} \equiv \mathbb{1}\right)$ and the $D_{i}(t)$ are some diagonal matrices that are expressed as exponential sums, i.e.,

$$
D_{i}(t)=\sum_{k=1}^{K} \exp \left(\Lambda_{i}^{(k)} t\right) D_{i}^{(k)} .
$$

Here $\Lambda_{i}^{(k)}$ and $D_{i}^{(k)}$ are some complex diagonal matrices. Partition $U_{I}(T)$ into $r$ segments $U_{I}\left(T, t_{r-1}\right) \cdots U_{I}\left(t_{1}, 0\right)$, 
whose respective durations $\Delta t_{w}, w=0, \ldots, r-1$, are determined by the partitioning scheme given in Sec. III B and the time markers $t_{w}$ are defined as $t_{w}=\sum_{l=0}^{w-1} \Delta t_{l}$. The total number of steps is denoted as $r$. The evolution operator from $t_{w}$ to $t_{w}+\Delta t_{w}$ is expressed as

$$
\begin{aligned}
U_{I}\left(t_{w}+\Delta t_{w}, t_{w}\right)= & \sum_{q=0}^{\infty} \sum_{\mathbf{i}_{q}} \sum_{\mathbf{k}_{q}} \sum_{x= \pm}(-i)^{q} \\
& \times \frac{\left[\left(e^{\Delta t_{w} \lambda}-1\right) / \lambda\right]^{q}}{2 q !} \Gamma_{\mathbf{i}_{q}}^{\left(\mathbf{k}_{q}\right)}\left(t_{w}\right) P_{\mathbf{i}_{q}} \Phi_{\mathbf{i}_{q}, x}^{\left(\mathbf{k}_{q}, w\right)}
\end{aligned}
$$

with

$$
\Gamma_{\mathbf{i}_{q}}^{\left(\mathbf{k}_{q}\right)}\left(t_{w}\right)=\left\|D_{i_{q}}^{\left(k_{q}\right)}\right\|_{\max } e^{t_{w} \lambda_{\left(i_{q}, k_{q}\right)}} \cdots\left\|D_{i_{1}}^{\left(k_{1}\right)}\right\|_{\max } e^{t_{w} \lambda_{\left(i_{1}, k_{1}\right)}},
$$

where $\|\cdot\|_{\max }$ is the $\max$ norm, $\lambda_{(i, k)}=\max _{z} \operatorname{Re}$ $\left(\left\langle z\left|\Lambda_{i}^{(k)}\right| z\right\rangle\right)$ is the maximum real part of $\Lambda_{i}^{(k)}$, and $\lambda=$ $\max _{i, k}\left\{\lambda_{(i, k)}\right\}$. Here, $\Phi_{\mathbf{i}_{q}, \pm}^{\left(\mathbf{k}_{q}, w\right)}$ are some diagonal unitaries as derived later in Eq. (38) and each $P_{\mathbf{i}_{q}}$ is a unique product of permutations. Note that the above evolution operators are given as a LCU. We provide a review for the LCU method in Appendix C. We set the truncation order $Q$ to be

$$
Q=\mathcal{O}\left(\frac{\log (r / \epsilon)}{\log \log (r / \epsilon)}\right)
$$

where $\epsilon$ is the overall simulation accuracy.

To implement the LCU routine for each $U_{I}\left(t_{w}+\right.$ $\left.\Delta t_{w}, t_{w}\right)$, we require preparing a state

$$
\begin{aligned}
\left|\psi_{0}\right\rangle & =\frac{1}{\sqrt{s}} \sum_{q=0}^{Q} \sum_{\mathbf{i}_{q}} \sum_{\mathbf{k}_{q}} \sum_{x=0,1} \\
\times & \sqrt{\frac{\left[\left(e^{\Delta t_{w} \lambda}-1\right) / \lambda\right]^{q} \Gamma_{\mathbf{i}_{q}}^{\left(\mathbf{k}_{q}\right)}\left(t_{w}\right)}{2 q !}}\left|\mathbf{i}_{q}\right\rangle\left|\mathbf{k}_{q}\right\rangle|x\rangle,
\end{aligned}
$$

where $\left|\mathbf{i}_{q}\right\rangle$ represents $Q$ quantum registers that each has dimension $M$ and $\left|\mathbf{k}_{q}\right\rangle$ represents $Q$ quantum registers that each has dimension $K$, and $s$ is the normalization factor. Following the same notation as used in Appendix C, let us denote the state preparation unitary as $B$, i.e., $B|0\rangle^{\otimes 2 Q+1}=$ $\left|\psi_{0}\right\rangle$ ( $B$ is explicitly given in Sec. III C 2). Let us denote by $V_{c}$ the control unitary such that

$$
V_{c}\left|\mathbf{i}_{q}\right\rangle\left|\mathbf{k}_{q}\right\rangle|x\rangle|\psi\rangle=\left|\mathbf{i}_{q}\right\rangle\left|\mathbf{k}_{q}\right\rangle|x\rangle(-i)^{q} P_{\mathbf{i}_{q}} \Phi_{\mathbf{i}_{q}, x}^{\left(\mathbf{k}_{q}, w\right)}|\psi\rangle .
$$

The oblivious amplitude amplification (OAA) involves interleaving the operator $W=\left(B^{\dagger} \otimes I\right) V_{c}(B \otimes I)$ as

$$
A=-W R W^{\dagger} R W,
$$

where $R \equiv I-2(|0\rangle\langle 0| \otimes I)$. For each piece of the unitary, we implement $A$ on the extended system $|0\rangle^{\otimes(2 Q+1)}|\psi\rangle$. By construction, we have

$$
\begin{aligned}
& \| A|0\rangle^{\otimes(2 Q+1)}|\psi\rangle-|0\rangle^{\otimes(2 Q+1)} U_{I}\left(t_{w}+\Delta t_{w}, t_{w}\right)|\psi\rangle \| \\
& \quad=\mathcal{O}\left(\frac{\epsilon}{r}\right) .
\end{aligned}
$$

This means that applying $A$ effectively performs the unitary $U_{I}\left(t_{w}+\Delta t_{w}, t_{w}\right)$ on the main system $|\psi\rangle$, with error $\mathcal{O}(\epsilon / r)$. Combining $r$ pieces of the procedure, it effectively simulates $U_{I}(T)$ with overall error $\mathcal{O}(\epsilon)$, i.e.,

$$
\begin{aligned}
\| A_{r-1} & \cdots A_{1} A_{0}|0\rangle^{\otimes(2 Q+1)}|\psi\rangle-|0\rangle^{\otimes(2 Q+1)} U_{I}(T)|\psi\rangle|| \\
& =\mathcal{O}(\epsilon),
\end{aligned}
$$

where $A_{w}$ are the OAA operators for the corresponding piece of evolution. This implies that applying the sequence of $A$ 's followed by the circuit for $e^{-i H_{0} T}$ can approach the action of $U(T)$ to an arbitrary accuracy.

\section{B. Permutation expansion for $U_{I}(t)$}

In this section, we give a thorough introduction of the permutation expansion in the Dyson series and the conditions that arise from implementing the LCU method. We focus on addressing the interaction-picture unitary $U_{I}(t)$, i.e., the time-ordered operator in Eq. (13). Using the expansions introduced in Eqs. (15) and (16), we get

$$
\begin{aligned}
U_{I}(t) & \equiv \mathcal{T} \exp \left[-i \int_{0}^{t} e^{i H_{0} t^{\prime}} V\left(t^{\prime}\right) e^{-i H_{0} t^{\prime}} d t^{\prime}\right] \\
& =\sum_{q=0}^{\infty}(-i)^{q} \int_{0}^{t} d \tau_{q} \cdots \int_{0}^{\tau_{2}} d \tau_{1} e^{i H_{0} \tau_{q}} V\left(\tau_{q}\right) e^{-i H_{0} \tau_{q}} \ldots \\
& \times e^{i H_{0} \tau_{1}} V\left(\tau_{1}\right) e^{-i H_{0} \tau_{1}} .
\end{aligned}
$$

We denote the basis in which $H_{0}$ is diagonal by $\{|z\rangle\}$ and its diagonal elements by $E_{z}=\left\langle z\left|H_{0}\right| z\right\rangle$. The action of $U_{I}(t)$ on a basis vector $|z\rangle$ becomes

$$
\begin{aligned}
U_{I}(t)|z\rangle= & \sum_{q=0}^{\infty} \sum_{\mathbf{i}_{q}} \sum_{\mathbf{k}_{q}}(-i)^{q} \int_{0}^{t} d \tau_{q} \cdots \\
& \times \int_{0}^{\tau_{2}} d \tau_{1} \exp \left[\left(i E_{z_{\mathbf{i}_{q}}}-i E_{z_{\mathbf{i}_{q-1}}}+\lambda_{i_{q}, \mathbf{i}_{q}}^{\left(k_{q}\right)}\right) \tau_{q}+\cdots\right. \\
& \left.+\left(i E_{z_{\mathbf{i}_{1}}}-i E_{z}+\lambda_{i_{1}, \mathbf{z}_{1}}^{\left(k_{1}\right)}\right) \tau_{1}\right] d_{\mathbf{i}_{q}, z}^{\left(\mathbf{k}_{q}\right)} P_{\mathbf{i}_{q}}|z\rangle
\end{aligned}
$$

where $E_{z_{\mathbf{i}_{j}}}$ is the $z_{\mathbf{i}_{j}}$ th diagonal element of $H_{0}$, i.e., $E_{z_{\mathbf{i}_{j}}}=$ $\left\langle z_{\mathbf{i}_{j}}\left|H_{0}\right| z_{\mathbf{i}_{j}}\right\rangle$, and $\left|z_{\mathbf{i}_{j}}\right\rangle=P_{\mathbf{i}_{j}}|z\rangle$ with $P_{\mathbf{i}_{j}}=P_{i_{j}} \cdots P_{i_{1}}$. By 
Identity 1 , this can be further simplified as

$$
U_{I}(t)|z\rangle=\sum_{q=0}^{\infty} \sum_{\mathbf{i}_{q}} \sum_{\mathbf{k}_{q}}(-i)^{q} e^{t\left[x_{1}, \ldots, x_{q}, 0\right]} d_{\mathbf{i}_{q}, z}^{\left(\mathbf{k}_{q}\right)} P_{\mathbf{i}_{q}}|z\rangle,
$$

where

$$
x_{j}=i\left(E_{z_{\mathbf{i}_{q}}}-E_{z_{\mathbf{i}_{j-1}}}\right)+\sum_{l=j}^{q} \lambda_{i_{l}, \mathrm{z}_{\mathbf{i}_{l}}}^{\left(k_{l}\right)} .
$$

\section{The $L C U$ routine}

To implement the LCU method for a quantum simulation of $U_{I}(T)$, we first decompose the overall simulation duration $T$ into $r$ pieces in sequence, i.e.,

$$
\begin{aligned}
U_{I}(T) & =U_{I}\left(T, t_{r-1}\right) U_{I}\left(t_{r-1}, t_{r-2}\right) \cdots U_{I}\left(t_{1}, 0\right) \\
& =\prod_{w=0}^{r-1} U_{I}\left(t_{w}+\Delta t_{w}, t_{w}\right),
\end{aligned}
$$

where the operators in the product of the last equation are understood to be ordered, $t_{w+1}=t_{w}+\Delta t_{w}$ and $t_{0} \equiv 0$ and $t_{r} \equiv T$. The number of steps, $r$, and the step size, $\Delta t_{w}$, are to be determined. When acting on a computational basis state, each piece in the decomposition can be written as

$$
\begin{aligned}
U_{I}( & \left.t_{w}+\Delta t_{w}, t_{w}\right)|z\rangle \\
= & \mathcal{T} \exp \left[-i \int_{t_{w}}^{t_{w}+\Delta t_{w}} H_{I}\left(t^{\prime}\right) d t^{\prime}\right]|z\rangle \\
= & \sum_{q=0}^{\infty} \sum_{\mathbf{i}_{q}} \sum_{\mathbf{k}_{q}}(-i)^{q} \int_{t_{w}}^{t_{w}+\Delta t_{w}} d \tau_{q} \ldots \\
& \times \int_{t_{w}}^{\tau_{2}} d \tau_{1} \exp \left[\sum_{l=1}^{q}\left(i E_{z_{\mathbf{i}_{l}}}-i E_{z_{\mathbf{i}_{l}-1}}+\lambda_{i_{l}, \mathbf{i}_{l}}^{\left(k_{l}\right)}\right) \tau_{l}\right] \\
& \times d_{\mathbf{i}_{q}, z}^{\left(\mathbf{k}_{q}\right)} P_{\mathbf{i}_{q}}|z\rangle, \\
= & \sum_{q=0}^{\infty} \sum_{\mathbf{i}_{q}} \sum_{\mathbf{k}_{q}}(-i)^{q} \exp \left[t_{w} \sum_{l=1}^{q}\left(i E_{z_{\mathbf{i}_{l}}}-i E_{z_{\mathbf{i}_{l}-1}}+\lambda_{i_{l}, \mathbf{z}_{l}}^{\left(k_{l}\right)}\right)\right] \\
& \times \int_{0}^{\Delta t_{w}} d \tau_{q}^{\prime} \ldots \\
& \times \int_{0}^{\tau_{2}^{\prime}} d \tau_{1}^{\prime} \exp \left[\sum_{l=1}^{q}\left(i E_{z_{\mathbf{i}_{l}}}-i E_{z_{\mathbf{i}_{l}-1}}+\lambda_{i_{l}, \mathbf{i}_{l} l}^{\left(k_{l}\right)}\right) \tau_{l}^{\prime}\right] \\
& \times d_{\mathbf{i}_{q}, z}^{\left(\mathbf{k}_{q}\right)} P_{\mathbf{i}_{q}}|z\rangle
\end{aligned}
$$

$$
\begin{aligned}
& =\sum_{q=0}^{\infty} \sum_{\mathbf{i}_{q}} \sum_{\mathbf{k}_{q}}(-i)^{q} \exp \left[t_{w} \sum_{l=1}^{q}\left(i E_{z_{\mathbf{i}_{l}}}-i E_{z_{\mathbf{i}_{l}-1}}+\lambda_{i_{l}, \mathbf{i}_{l}}^{\left(k_{l}\right)}\right)\right] \\
& \times e^{\Delta t_{w}\left[x_{1}, x_{2}, \ldots, x_{q}, 0\right]} d_{\mathbf{i}_{q, z}}^{\left(\mathbf{k}_{q}\right)} P_{\mathbf{i}_{q}}|z\rangle \\
& =\sum_{q=0}^{\infty} \sum_{\mathbf{i}_{q}} \sum_{\mathbf{k}_{q}}(-i)^{q} e^{-i t_{w}\left(E_{\mathbf{z}_{0}}-E_{\mathbf{i}_{q}}\right)} e^{t_{w} \sum_{l=1}^{q} \lambda_{i l, \mathbf{i}_{l}}^{\left(k_{i}\right)}} \\
& \times e^{\Delta t_{w}\left[x_{1}, x_{2}, \ldots, x_{q}, 0\right]} d_{\mathbf{i}_{q}, z}^{\left(\mathbf{k}_{q}\right)} P_{\mathbf{i}_{q}}|z\rangle,
\end{aligned}
$$

which has the same form as Eq. (27) except that the integration intervals are shifted (with $E_{z_{\mathbf{i}_{0}}} \equiv E_{z}$ ). We can set

$$
d_{\mathbf{i}_{q}, z}^{\left(\mathbf{k}_{q}\right)}\left(t_{w}\right)=d_{\mathbf{i}_{q}, z}^{\left(\mathbf{k}_{q}\right)} e^{t_{w} \sum_{l=1}^{q} \lambda_{i_{l}, \mathbf{i}_{l}}^{\left(k_{l}\right)}}
$$

which leads to

$$
\begin{aligned}
U_{I}\left(t_{w}+\Delta t_{w}, t_{w}\right)|z\rangle & =\sum_{q=0}^{\infty} \sum_{\mathbf{i}_{q}} \sum_{\mathbf{k}_{q}}(-i)^{q} e^{-i t_{w}\left(E_{z_{\mathbf{i}_{0}}}-E_{z_{\mathbf{i}_{q}}}\right)} \\
& \times e^{\Delta t_{w}\left[x_{1}, x_{2}, \ldots, x_{q}, 0\right]} d_{\mathbf{i}_{q}, z}^{\left(\mathbf{k}_{q}\right)}\left(t_{w}\right) P_{\mathbf{i}_{q}}|z\rangle .
\end{aligned}
$$

To formulate the above expression in terms of a linear combination of unitaries, we need to evaluate the norms of $e^{\Delta t_{w}\left[x_{1}, x_{2}, \ldots, x_{q}, 0\right]}$ and $d_{\mathbf{i}_{q}, z}^{\left(\mathbf{k}_{q}\right)}\left(t_{w}\right)$. The norm of $d_{\mathbf{i}_{q}, z}^{\left(\mathbf{k}_{q}\right)}\left(t_{w}\right)$ is bounded by

$$
\begin{aligned}
\left|d_{\mathbf{i}_{q}, z}^{\left(\mathbf{k}_{q}\right)}\left(t_{w}\right)\right| & \leq\left.\left.\left\|D_{i_{q}}^{\left(k_{q}\right)}\right\|\right|_{\max } e^{t_{w} \lambda_{\left(i_{q}, k_{q}\right)}} \cdots\left\|D_{i_{1}}^{\left(k_{1}\right)}\right\|\right|_{\max } e^{t_{w} \lambda_{\left(i_{1}, k_{1}\right)}} \\
& =\Gamma_{\mathbf{i}_{q}}^{\left(\mathbf{k}_{q}\right)}\left(t_{w}\right)
\end{aligned}
$$

The norm of the $e^{\Delta t_{w}\left[x_{1}, x_{2}, \ldots, x_{q}, 0\right]}$ can be bounded by using the following identity.

Identity 2. For any $q+1$ complex values $x_{0}, \ldots, x_{q} \in \mathbb{C}$,

$$
\left|e^{\left[x_{0}, \ldots, x_{q}\right]}\right| \leq e^{\left[\operatorname{Re}\left(x_{0}\right), \ldots, \operatorname{Re}\left(x_{q}\right)\right]}=\frac{e^{\xi}}{q !},
$$

where $\operatorname{Re}(\cdot)$ denotes the real part of an input and $\xi \in$ $\left[\min \left\{\operatorname{Re}\left(x_{0}\right), \ldots, \operatorname{Re}\left(x_{q}\right)\right\}, \max \left\{\operatorname{Re}\left(x_{0}\right), \ldots, \operatorname{Re}\left(x_{q}\right)\right\}\right]$.

The proof can be found in Appendix A. From Identity 2, we show in Appendix B that

$$
\begin{aligned}
\left|e^{\Delta t_{w}\left[x_{1}, x_{2}, \ldots, x_{q}, 0\right]}\right| & \leq e^{\Delta t_{w}[q \lambda,(q-1) \lambda, \ldots, \lambda, 0]} \\
& =\frac{1}{q !}\left(\frac{e^{\Delta t_{w} \lambda}-1}{\lambda}\right)^{q} \\
& \equiv \frac{\widetilde{\Delta t_{w}^{q}}}{q !},
\end{aligned}
$$


where we have defined the quantity

$$
\widetilde{\Delta t_{w}} \equiv \frac{e^{\Delta t_{w} \lambda}-1}{\lambda} .
$$

With these bounds, the factors in the expansion form in Eq. (31) can be written as

$$
\begin{aligned}
e^{\Delta t_{w}\left[x_{1}, x_{2}, \ldots, x_{q}, 0\right]} d_{\mathbf{i}_{q}, z}^{\left(\mathbf{k}_{q}\right)}\left(t_{w}\right) \\
=\frac{\widetilde{\Delta t_{w}^{q}}}{q !} \Gamma_{\mathbf{i}_{q}}^{\left(\mathbf{k}_{q}\right)}\left(t_{w}\right) \cos \left[\phi_{\mathbf{i}_{q}, z}^{\left(\mathbf{k}_{q}\right)}\right] e^{i \theta_{\mathbf{i}_{q}, z}^{\left(\mathbf{k}_{q}\right)}} \\
=\frac{\widetilde{\Delta t}}{2 q !} \Gamma_{\mathbf{i}_{q}}^{\left(\mathbf{k}_{q}\right)}\left(t_{w}\right)\left(e^{i \phi_{\mathbf{i}_{q}, z}^{\left(\mathbf{k}_{q}\right)}+i \theta_{\mathbf{i}_{q}, z}^{\left(\mathbf{k}_{q}\right)}}+e^{-i \phi_{\mathbf{i}_{q}, z}^{\left(\mathbf{k}_{q}\right)}+i \theta_{\mathbf{i}_{q}, z}^{\left(\mathbf{k}_{q}\right)}}\right),
\end{aligned}
$$

where

$$
\phi_{\mathbf{i}_{q}, z}^{\left(\mathbf{k}_{q}\right)}=\cos ^{-1}\left[\left|\frac{e^{\Delta t_{w}\left[x_{1}, x_{2}, \ldots, x_{q}, 0\right]} d_{\mathbf{i}_{q}, z}^{\left(\mathbf{k}_{q}\right)}\left(t_{w}\right)}{\left(\widetilde{\Delta t_{w}^{q}} / q !\right) \Gamma_{\mathbf{i}_{q}}^{\left(\mathbf{k}_{q}\right)}\left(t_{w}\right)}\right|\right]
$$

and

$$
\theta_{\mathbf{i}_{q}, z}^{\left(\mathbf{k}_{q}\right)}=\arg \left[\frac{e^{\Delta t_{w}\left[x_{1}, x_{2}, \ldots, x_{q}, 0\right]} d_{\mathbf{i}_{q}, z}^{\left(\mathbf{k}_{q}\right)}\left(t_{w}\right)}{\left(\widetilde{\Delta t_{w}^{q}} / q !\right) \Gamma_{\mathbf{i}_{q}}^{\left(\mathbf{k}_{q}\right)}\left(t_{w}\right)}\right] .
$$

The evolution operator from $t_{w}$ to $t_{w}+\Delta t_{w}$ becomes

$$
\begin{aligned}
U_{I}\left(t_{w}+\Delta t_{w}, t_{w}\right)=\sum_{z} U_{I}\left(t_{w}+\Delta t_{w}, t_{w}\right)|z\rangle\langle z| \\
=\sum_{z} \sum_{q=0}^{\infty} \sum_{\mathbf{i}_{q}} \sum_{\mathbf{k}_{q}}(-i)^{q} \frac{\widetilde{\Delta t_{w}}}{2 q !} \Gamma_{\mathbf{i}_{q}}^{\left(\mathbf{k}_{q}\right)}\left(t_{w}\right) \\
\quad \times\left(e^{i \phi_{\mathbf{i}_{q}, z}^{\left(\mathbf{k}_{q}\right)}+i i_{\mathbf{i}_{q}, z}^{\left(\mathbf{k}_{q}\right)}}+e^{\left.-i \phi_{\mathbf{i}_{q}, z}^{\left(\mathbf{k}_{q}\right)}+i \theta_{\mathbf{i}_{q}, z}^{\left(\mathbf{k}_{q}\right)}\right) P_{\mathbf{i}_{q}}|z\rangle\langle z|}\right. \\
=\sum_{q=0}^{\infty} \sum_{\mathbf{i}_{q}} \sum_{\mathbf{k}_{q}} \sum_{x= \pm}(-i)^{q} \frac{\widetilde{\Delta t_{w}^{q}}}{2 q !} \Gamma_{\mathbf{i}_{q}}^{\left(\mathbf{k}_{q}\right)}\left(t_{w}\right) P_{\mathbf{i}_{q}} \Phi_{\mathbf{i}_{q}, x}^{\left(\mathbf{k}_{q}, w\right)},
\end{aligned}
$$

where $\Phi_{\mathbf{i}_{q}, \pm}^{\left(\mathbf{k}_{q}, w\right)}$ are diagonal unitaries with diagonal elements being $e^{i\left( \pm \phi_{\mathbf{i}_{q}, z}^{(\mathbf{k} q)}+\theta_{\mathbf{i}_{q, z}}^{\left(\mathbf{k}_{q}\right)} \text {. }\right.}$.

To implement the LCU method for simulating $U_{I}\left(t_{w}+\right.$ $\left.\Delta t_{w}, t_{w}\right)$, we require a preparation of the state

$$
\begin{aligned}
\left|\psi_{0}\right\rangle= & \frac{1}{\sqrt{s}} \sum_{q=0}^{Q} \sum_{\mathbf{i}_{q}} \sum_{\mathbf{k}_{q}} \sum_{x=0,1} \sqrt{\frac{\widetilde{\Delta t_{w}^{q}}}{2 q !} \Gamma_{\mathbf{i}_{q}}^{\left(\mathbf{k}_{q}\right)}\left(t_{w}\right)}\left|i_{1}\right\rangle \\
& \cdots\left|i_{q}\right\rangle \otimes|0\rangle^{\otimes(Q-q)}\left|k_{1}\right\rangle \cdots\left|k_{q}\right\rangle \otimes|0\rangle^{\otimes(Q-q)}|x\rangle \\
\equiv & \frac{1}{\sqrt{s}} \sum_{q=0}^{Q} \sum_{\mathbf{i}_{q}} \sum_{\mathbf{k}_{q}} \sum_{x=0,1} \sqrt{\frac{\widetilde{\Delta t_{w}^{q}}}{2 q !} \Gamma_{\mathbf{i}_{q}}^{\left(\mathbf{k}_{q}\right)}\left(t_{w}\right)\left|\mathbf{i}_{q}\right\rangle\left|\mathbf{k}_{q}\right\rangle|x\rangle}
\end{aligned}
$$

where $\left|\mathbf{i}_{q}\right\rangle$ represents $Q$ quantum registers that each has dimension $M$ and $\left|\mathbf{k}_{q}\right\rangle$ represents $Q$ quantum registers that each has dimension $K$. The normalization constant is

$$
s=\sum_{q=0}^{Q} \sum_{\mathbf{i}_{q}} \sum_{\mathbf{k}_{q}} \sum_{x=0,1} \frac{\widetilde{\Delta t_{w}^{q}}}{2 q !} \Gamma_{\mathbf{i}_{q}}^{\left(\mathbf{k}_{q}\right)}\left(t_{w}\right) \equiv \sum_{q=0}^{Q} \frac{\left[\Gamma\left(t_{w}\right) \widetilde{\Delta t_{w}}\right]^{q}}{q !}
$$

where we have defined

$$
\Gamma\left(t_{w}\right) \equiv \sum_{i=0}^{M} \sum_{k=1}^{K}\left\|D_{i}^{(k)}\right\| \|_{\max } e^{t_{w} \lambda_{(i, k)}}
$$

and we note that $\Gamma\left(t_{w}\right)$ is an upper bound on the maxnorm of the interaction Hamiltonian at time $t_{w}, \Gamma\left(t_{w}\right) \geq$ $\left\|V\left(t_{w}\right)\right\|_{\max }$. The quantity $\Gamma\left(t_{w}\right)$ is related to the energy strength in a typical LCU setup [14]. In Appendix D, we provide an alternative way that uses a larger bound $\Gamma=M K \max _{\forall k, i}|| D_{i}^{(k)} \|_{\max }$, which leads to an exponential saving for the state preparation. We proceed with $\Gamma\left(t_{w}\right)$ hereafter.

The OAA step in the LCU method requires $s \approx 2$. This leads to

$$
\Gamma\left(t_{w}\right) \widetilde{\Delta t_{w}}=\Gamma\left(t_{w}\right) \frac{e^{\Delta t_{w} \lambda}-1}{\lambda}=\ln 2,
$$

and Eq. (40) becomes a truncated Taylor expansion of 2 up to order $Q$, i.e., $2 \approx \sum_{q=0}^{Q}(\ln 2)^{q} / q$ !. If we require $\mid s-$ $2 \mid \leq \epsilon / r$, where $r$ is the total number of steps and $\epsilon$ is some positive number, then the simulation error for each $U_{I}\left(t_{w}+\right.$ $\left.\Delta t_{w}, t_{w}\right)$ is also within $\epsilon / r$. The required truncation order with this accuracy scales as

$$
Q=\mathcal{O}\left(\frac{\log (r / \epsilon)}{\log \log (r / \epsilon)}\right)
$$

\section{Time partitioning and the number of time steps}

The condition in Eq. (42) imposes a constraint on the next step size $\Delta t_{w}$ given the current time $t_{w}$,

$$
\Delta t_{w}=\frac{1}{\lambda} \ln \left(1+\frac{\lambda}{\Gamma\left(t_{w}\right)} \ln 2\right) .
$$

Remembering that $\Gamma\left(t_{w}\right)$ is a function of $t_{w}=\sum_{l=0}^{w-1} \Delta t_{l}$, this condition determines the schedule, as every $\Delta t_{w}$ is determined by the preceding time steps.

Special care should be given when setting the last time step, as $\Delta t_{w}$ can become too large that it exceeds the total desired evolution time $T$. Whenever $t_{w+1}$ is found to be greater than $T$ [or if the argument inside the $\ln (\cdot)$ is found to be negative], one should replace the bound $\Gamma\left(t_{w}\right)$ with a larger bound $\tilde{\Gamma}\left(t_{w}\right)=\lambda \ln 2 /\left(e^{\lambda \Delta t_{w}}-1\right)$ and set the final step $\Delta t_{w}=T-t_{w}$. 
Let us now examine the dependence of $\Delta t_{w}$ on $\Gamma\left(t_{w}\right)$ in order to determine a bound on the number of time steps (equivalently, number of repetitions) $r$ required for the execution of the entire time evolution. We distinguish between three cases. (i) When $\lambda=0$, we have $\Delta t_{w} \Gamma\left(t_{w}\right)=\ln 2$, similar to the time-independent case, though we note that a vanishing maximal $\lambda$ could imply time-dependent oscillations as well. This can be seen by taking the $\lambda \rightarrow 0$ limit of Eq. (44). (ii) In the case where $\lambda<0$, i.e., a system with a decaying $\Gamma\left(t_{w}\right)$, we have $\Delta t_{w} \Gamma\left(t_{w}\right) \geq \ln 2$, i.e., the time steps are longer than $\ln 2 / \Gamma\left(t_{w}\right)$. Furthermore, the total number of steps $r$ is finite even for an arbitrarily large evolution time $T$. Note that, since $\Gamma\left(t_{w}\right)$ approaches zero asymptotically, for a large enough time $t_{w *}$, we have $\Gamma\left(t_{w *}\right)<|\lambda| \ln 2$, i.e., the argument inside the logarithm above becomes negative. This indicates it reaches the final step, i.e., the bound should be modified as $\tilde{\Gamma}\left(t_{w *}\right)=\lambda \ln 2 /\left(e^{\lambda \Delta t_{w *}}-1\right)$ and $\Delta t_{w *}=T-t_{w *}$ becomes the final step. (iii) In the case where $\lambda>0$ [an amplified $\Gamma\left(t_{w}\right)$ ], we have $\Gamma\left(t_{w}\right) \gg \lambda$ at large simulation times, $t_{w}$. From Eq. (44), we have $\Delta t_{w} \rightarrow \ln 2 / \Gamma\left(t_{w}\right)$ in this limit.

We see that (for large enough simulation times) the time step $\Delta t_{w}$ is inversely proportional to $\Gamma\left(t_{w}\right)$ that upper bounds the max-norm of the interaction Hamiltonian at time $t_{w}$. Therefore, we have $\sum_{w=0}^{r-1} \Gamma\left(t_{w}\right) \Delta t_{w} \gtrsim r \ln 2$, which implies that $r \lesssim \sum_{w=0}^{r-1} \Gamma\left(t_{w}\right) \Delta t_{w} / \ln 2$.

It would be instructive to compare the above scaling with that of Ref. [17] in which the simulation algorithm is said to have an $L^{1}$-norm scaling, i.e., an algorithm cost scaling linearly with $\int_{0}^{t} d \tau H_{\max }(\tau)$ up to logarithmic factors. Under a similar intuition, our algorithm has a discretized $L^{1}$-norm-like scaling with $\sum_{w=0}^{r-1} \Gamma\left(t_{w}\right) \Delta t_{w}$. However, in our case, $\Gamma\left(t_{w}\right)$ is related to the norm of the interaction Hamiltonian.

\section{State preparation}

In this subsection, we provide a procedure to prepare the state $\left|\psi_{0}\right\rangle$ given in Eq. (39). First, we initialize a state $|0\rangle^{\otimes Q}|0\rangle^{\otimes Q}|0\rangle$, where each of the first $Q$ registers has dimension $M$ (responsible for the $\left|\mathbf{i}_{q}\right\rangle$ part), each of the later $Q$ registers has dimension $K$ (responsible for the $\left|\mathbf{k}_{q}\right\rangle$ part), and the last register is a qubit (for the cosine decomposition). For simplicity, we can perform a Hadamard gate on the last qubit and then omit its dependence for the following discussion. The next step is to create a state of the form

$$
\frac{1}{\sqrt{s}} \sum_{q=0}^{Q} \sqrt{s_{q}}|1\rangle^{\otimes q}|0\rangle^{\otimes(Q-q)}|1\rangle^{\otimes q}|0\rangle^{\otimes(Q-q)},
$$

where $s_{q} \equiv\left[\Gamma\left(t_{w}\right) \widetilde{\Delta t_{w}}\right]^{q} / q$ !. For each $|1\rangle$ from the first $Q$ registers (the $\left|\mathbf{i}_{q}\right\rangle$ part) and the corresponding $|1\rangle$ in the later $Q$ registers (the $\left|\mathbf{k}_{q}\right\rangle$ part), we make

$$
|1\rangle|1\rangle \rightarrow \sum_{i=0}^{M} \sum_{k=1}^{K} \sqrt{\frac{\left\|D_{i}^{(k)}\right\| \|_{\max } e^{t_{w} \lambda_{(i, k)}}}{\Gamma\left(t_{w}\right)}}|i\rangle|k\rangle .
$$

Then Eq. (45) becomes

$$
\begin{aligned}
& \frac{1}{\sqrt{s}} \sum_{q=0}^{Q} \sqrt{s_{q}} \sum_{\mathbf{i}_{q}} \sum_{\mathbf{k}_{q}} \sqrt{\frac{\Gamma_{\mathbf{i}_{q}}^{\left(\mathbf{k}_{q}\right)}\left(t_{w}\right)}{\left[\Gamma\left(t_{w}\right)\right]^{q}}}\left|\mathbf{i}_{q}\right\rangle\left|\mathbf{k}_{q}\right\rangle \\
& =\frac{1}{\sqrt{s}} \sum_{q=0}^{Q} \sum_{\mathbf{i}_{q}} \sum_{\mathbf{k}_{q}} \sqrt{\frac{\left(\widetilde{\left.\Delta t_{w}\right)^{q}}\right.}{q !} \Gamma_{\mathbf{i}_{q}}^{\left(\mathbf{k}_{q}\right)}\left(t_{w}\right)}\left|\mathbf{i}_{q}\right\rangle\left|\mathbf{k}_{q}\right\rangle,
\end{aligned}
$$

which is the required $\left|\psi_{0}\right\rangle$ in Eq. (39), when combined with $|x\rangle$.

Next, we provide a process that produces the state in Eq. (45). First, we perform a rotation that takes the first register in the $\left|\mathbf{i}_{q}\right\rangle$ part to

$$
|0\rangle \rightarrow \frac{1}{\sqrt{s}}\left(|0\rangle+\sqrt{\sum_{q=1}^{Q} s_{q}|1\rangle}\right),
$$

and perform a control gate from the first register to the second (both in the $\left|\mathbf{i}_{q}\right\rangle$ part) such that

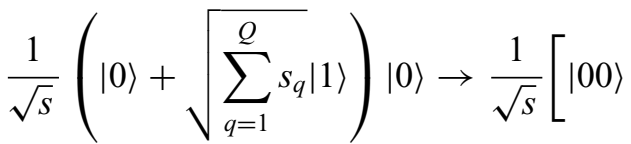

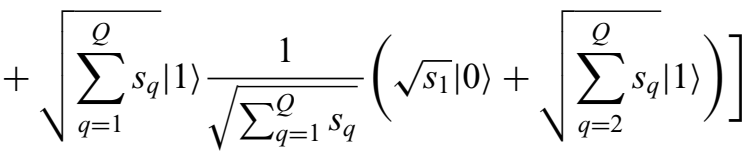

$$
\begin{aligned}
& =\frac{1}{\sqrt{s}}\left(|00\rangle+\sqrt{s_{1}}|10\rangle+\sqrt{\sum_{q=2}^{Q} s_{q}}|11\rangle\right) \text {. }
\end{aligned}
$$

Continuing this procedure for the rest of the registers in the $\left|\mathbf{i}_{q}\right\rangle$ part, the state becomes

$$
|0\rangle^{\otimes Q} \rightarrow \frac{1}{\sqrt{s}} \sum_{q=0}^{Q} \sqrt{s_{q}}|1\rangle^{\otimes q}|0\rangle^{\otimes(Q-q)} .
$$

At this step, we perform controlled-NOT (CNOT) operations [35] from the first $Q$ registers ( $\left|\mathbf{i}_{q}\right\rangle$ part) to the last $Q$ registers ( $\left|\mathbf{k}_{q}\right\rangle$ part) correspondingly, e.g., perform a CNOT operation from the first register in the $\left|\mathbf{i}_{q}\right\rangle$ part to the first register in the $\left|\mathbf{k}_{q}\right\rangle$ part, and so on and so forth. Finally, we 
have

$$
\begin{aligned}
& \frac{1}{\sqrt{s}} \sum_{q=0}^{Q} \sqrt{s_{q}}|1\rangle^{\otimes q}|0\rangle^{\otimes(Q-q)}|0\rangle^{\otimes Q} \\
& \quad \rightarrow \frac{1}{\sqrt{s}} \sum_{q=0}^{Q} \sqrt{s_{q}}|1\rangle^{\otimes q}|0\rangle^{\otimes(Q-q)}|1\rangle^{\otimes q}|0\rangle^{\otimes(Q-q)},
\end{aligned}
$$

which gives Eq. (45), as required. The estimated gate cost for the preparation of $\left|\psi_{0}\right\rangle$ is $\mathcal{O}(Q M K)$. More detail regarding the cost is provided in Sec. III D 1.

\section{Implementation of the controlled unitaries}

The second ingredient of the LCU routine is the construction of the controlled operation

$$
V_{c}\left|\mathbf{i}_{q}\right\rangle\left|\mathbf{k}_{q}\right\rangle|x\rangle|\psi\rangle=\left|\mathbf{i}_{q}\right\rangle\left|\mathbf{k}_{q}\right\rangle|x\rangle(-i)^{q} P_{\mathbf{i}_{q}} \Phi_{\mathbf{i}_{q}, x}^{\left(\mathbf{k}_{q}, w\right)}|\psi\rangle .
$$

Taking an approach similar to that taken in Ref. [34], we first note that Eq. (52) indicates that $V_{c}$ can be carried out in two steps: a controlled-phase operation $\left(V_{c \Phi}\right)$ followed by a controlled-permutation operation $\left(V_{c P}\right)$.

The controlled-phase operation $V_{c \Phi}$ requires a somewhat intricate calculation of nontrivial phases. We therefore carry out the required algebra with the help of additional ancillary registers and then "push" the results into phases. The latter step is done by employing the unitary

$$
U_{\mathrm{ph}}|\varphi\rangle=e^{-i \varphi}|\varphi\rangle,
$$

whose implementation cost depends only on the precision with which we specify $\varphi$ and is independent of Hamiltonian parameters [33] (see Ref. [34] for a complete derivation). With the help of the (controlled) unitary transformation

$$
V_{\chi \phi}\left|\mathbf{i}_{q}\right\rangle\left|\mathbf{k}_{q}\right\rangle|x\rangle|z\rangle|0\rangle=\left|\mathbf{i}_{q}\right\rangle\left|\mathbf{k}_{q}\right\rangle|x\rangle|z\rangle\left|\chi_{\mathbf{i}_{q}}^{(z)}+(-1)^{k} \phi_{\mathbf{i}_{q}}^{(z)}\right\rangle,
$$

we can write $V_{c \Phi}=V_{\chi \phi}^{\dagger}\left(\mathbb{1} \otimes U_{\mathrm{ph}}\right) V_{\chi \phi}$, so that

$$
V_{c \Phi}\left|\mathbf{i}_{q}\right\rangle\left|\mathbf{k}_{q}\right\rangle|x\rangle|z\rangle=\left|\mathbf{i}_{q}\right\rangle\left|\mathbf{k}_{q}\right\rangle|x\rangle \Phi_{\mathbf{i}_{q}}^{(k)}|z\rangle .
$$

Note that $V_{\chi \phi}$ sends computational basis states to computational basis states. We provide an explicit construction of $V_{\chi \phi}$ in Ref. [34]. We find that its gate cost is $\mathcal{O}\left[Q M\left(k_{\mathrm{od}}+\log M\right)+Q M K\left(C_{D}+C_{\Delta H_{0}}+C_{\Lambda}\right)\right]$ and qubit cost is $\mathcal{O}[Q \log (M K)]$. Additional details are provided in Sec. III D 1.

The construction of $V_{c P}$ is carried out by a repeated execution of the simpler unitary transformation $\mathrm{U}_{p}|i\rangle|z\rangle=$ $|i\rangle P_{i}|z\rangle$. Recall that the $P_{i}$ are the off-diagonal permutation operators that appear in the Hamiltonian. The gate cost of $U_{p}$ is therefore $\mathcal{O}\left[M\left(k_{\mathrm{od}}+\log M\right)\right]$. Additional details may be found in Ref. [34].

\section{Algorithm cost}

We next analyze the circuit costs for the permutation expansion algorithm. Recall that the simulation of $U(T)$ consists of two operations $-e^{-i H_{0} T}$ and $U_{I}(T)$. The diagonal unitary $e^{-i H_{0} T}$ can be implemented efficiently with a gate cost that scales linearly with the system size. To observe this, note that $H_{0}$ is a diagonal matrix with real diagonal elements and can be written as $H_{0}=\sum_{\gamma=0}^{L} J_{\gamma} Z_{\gamma}$, where each $Z_{\gamma}$ is a tensor products of Pauli- $Z$ operators $(Z \otimes \cdots \otimes Z)$ acting on at most $d$ qubits (weight- $d$ operators). Hence, we can write $e^{-i H_{0} T}=\prod_{\gamma=0}^{L} e^{-i J_{\gamma} Z_{\gamma} T}$. Each $e^{-i J_{\gamma} Z_{\gamma} T}$ can be simulated using at most $2 d$ CNOT gates with a single ancillary qubit. For example, let $Z_{\gamma}$ be a weight- $m$ $(m \leq d)$ operator; then $e^{-i J_{\gamma} Z_{\gamma} T}$ can be implemented as

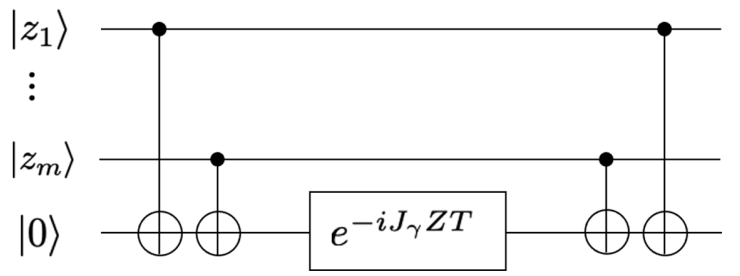

where $\left|z_{1}\right\rangle \cdots\left|z_{m}\right\rangle$ are the qubits $Z_{\gamma}$ acts on and $|0\rangle$ is an ancillary qubit for extracting the phase. There are total $L$ such implementations for $e^{-i H_{0} T}$. Therefore, the total gate cost is $\mathcal{O}(L d)$ and the qubit cost is $\mathcal{O}(1)$. Since $L$ usually grows linearly with the system size, the gate cost also scales linearly.

\section{The cost for the state preparation and the controlled unitaries}

The cost of implementing $U_{I}(T)$ resembles that in Ref. [34]. The first ingredient is the preparation of state $\left|\psi_{0}\right\rangle$. Recall from Sec. IIIC2 that the operation that takes $|0\rangle^{\otimes Q}|0\rangle^{\otimes Q}|0\rangle$ to $(1 / \sqrt{s}) \sum_{q=0}^{Q} \sqrt{s_{q}}|1\rangle^{\otimes q}$ $|0\rangle^{\otimes(Q-q)}|1\rangle^{\otimes q}|0\rangle^{\otimes(Q-q)}$ has gate cost $\mathcal{O}(Q)$. The operation for $|1\rangle|1\rangle \rightarrow \sum_{i=0}^{M} \sum_{k=1}^{K} \sqrt{\left[\left\|D_{i}^{(k)}\right\|_{\max } e^{t_{w} \lambda_{(i, k)}} / \Gamma\left(t_{w}\right)\right]}|i\rangle|k\rangle$ costs $\mathcal{O}(M K)[14,36]$. The total gate cost for the preparation of $\left|\psi_{0}\right\rangle$ (i.e., $\left.B\right)$ is $\mathcal{O}(Q M K)$. In Appendix $\mathrm{D}$, we provide an alternative procedure that leads to a $\mathcal{O}[Q \log (M K)]$ scaling for implementing $B$. The qubit cost in the state preparation is $\mathcal{O}[Q \log (M K)]$.

The next component is the implementation of the control unitary $V_{c}$. As shown in Ref. [34], the gate cost of performing the control permutation $P_{\mathbf{i}_{q}}$ is $\mathcal{O}\left[Q M\left(k_{\mathrm{od}}+\log M\right)\right]$, where $k_{\mathrm{od}}$ is the "locality," i.e., each permutation $P_{i}$ is a tensor product of at most $k_{\text {od }}$ Pauli- $X$ operators. The implementation of the control phase $\Phi_{\mathbf{i}_{q}, x}^{\left(\mathbf{k}_{q}, w\right)}$ involves the calculation of $d_{\mathbf{i}_{q}, z}^{\left(\mathbf{k}_{q}\right)}$ (the product of diagonal elements in the permutation expansion) and the divided differences (with the $x_{j}$ being the inputs). The cost of the former is $\mathcal{O}\left(Q M K C_{D}\right)$, where $C_{D}$ is the cost of obtaining an 
element of $D_{i}^{(k)}$. The cost of the latter is $\mathcal{O}\left[Q M\left(k_{\mathrm{od}}+\right.\right.$ $\left.\log M)+Q M K\left(C_{\Delta H_{0}}+C_{\Lambda}\right)\right]$, where $C_{\Delta H_{0}}\left(C_{\Lambda}\right)$ is the cost of obtaining energy differences of $H_{0}$ (elements of $\Lambda_{i}^{(k)}$ ) [therefore, $C_{\Delta H_{0}}+C_{\Lambda}$ is the cost for obtaining the inputs, $x_{j}$, as defined in Eq. (28)]. The additional cost for the reversibility of the process scales as $\mathcal{O}\left(Q^{2}\right)$. A detailed discussion of the costs of $C_{\Delta H_{0}}$ and $C_{\Lambda}$ may be found in Ref. [34]. Combining these, we estimate that the total cost for $V_{c}$ is

$$
\mathcal{O}\left[Q^{2}+Q M\left(k_{\mathrm{od}}+\log M\right)+Q M K\left(C_{D}+C_{\Delta H_{0}}+C_{\Lambda}\right)\right] .
$$

\section{Overall cost of the algorithm}

The full simulation for $U_{I}(T)$ is a product of segments $U_{I}\left(t_{w}+\Delta t_{w}, t_{w}\right)$, where each segment is simulated by interleaving $B$ and $V_{c}$. The total number of segments, $r$, is determined by $T=\sum_{w=0}^{r-1} \Delta t_{w}$, where each $\Delta t_{w}$ is determined by the partitioning scheme described in Sec. III C 1.

As discussed above, the number of LCU applications $r$ can be upper bounded by $r \lesssim \sum_{w=0}^{r-1} \Gamma\left(t_{w}\right) \Delta t_{w} / \ln 2$ (in the long simulation time limit), which can be viewed as a discretized $L^{1}$-norm-like scaling with the norm of the nonstatic component of the Hamiltonian $V(t)$.

Combining with the cost for simulating $e^{-i H_{0} T}$ with the cost for each step (56), we conclude that at worst the total gate cost scales as

$$
\begin{aligned}
& \mathcal{O}\left[r Q^{2}+r Q M\left(k_{\mathrm{od}}+\log M\right)\right. \\
& \left.+r Q M K\left(C_{D}+C_{\Delta H_{0}}+C_{\Lambda}\right)+L d\right],
\end{aligned}
$$

and the qubit cost scales as

$$
\mathcal{O}[Q \log (M K)],
$$

where $Q$ scales as $\mathcal{O}[\log (r / \epsilon) / \log \log (r / \epsilon)]$. A summary of the gate and qubit costs of the simulation circuit and the various subroutines used to construct it is given in Table I. A comparison to the best existing algorithms is also provided in Table II.

\section{Example advantages of the algorithm}

To illustrate how our simulation algorithm can provide speedups over existing algorithms, we focus in this subsection on two types of Hamiltonian system: highly oscillating systems and decaying systems.

The cost of our algorithm is independent of the oscillation rates of the dynamics, whereas the cost of any simulation algorithm (e.g., Refs. [12,15-17]) that depends on $\|d H(t) / d t\|$ would depend on oscillation rates of the system. To illustrate this advantage, consider a two-level system with a Hamiltonian

$$
H(t)=h Z+\Gamma\left(e^{-i \alpha t}|0\rangle\left\langle 1\left|+e^{i \alpha t}\right| 1\right\rangle\langle 0|\right)=H_{0}+V(t),
$$

where $h, \Gamma, \alpha \in \mathbb{R}, H_{0}=h Z$, and $V(t)=\Gamma\left(e^{-i \alpha t}|0\rangle\langle 1|+\right.$ $\left.e^{i \alpha t}|1\rangle\langle 0|\right)$. In this case, we have $k_{\mathrm{od}}=M=K=1$ and $\lambda=0$. The gate cost of simulating $U(T)$ scales as

$$
\mathcal{O}\left[\Gamma T\left(\frac{\log \Gamma T / \epsilon}{\log \log \Gamma T / \epsilon}\right)^{2}\right]
$$

which is independent of $\alpha$. This means that the simulation cost remains the same even if $\alpha$ becomes arbitrarily large. One can realize the absence of $\alpha$ owing to the fact that phases are explicitly integrated out into an integralfree expansion series, where the bound of each term does not depend on the oscillations (see Identity 2). Therefore, our simulation can be significantly more effective when the time dependence of the Hamiltonian has very high frequencies. Note that while the example above was given for a simple qubit system with pure oscillation, the frequency independence in cost holds for any system.

Another class of systems for which our algorithm can provide speedup are Hamiltonians with exponential decays, i.e., $\lambda<0$, which play an important role in many-body physics [37]. For concreteness, consider the Hamiltonian

$$
H(t)=h Z+\Gamma e^{-\alpha t} X=H_{0}+V(t),
$$

where $h, \Gamma \in \mathbb{R}, \alpha>0, H_{0}=h Z$, and $V(t)=\Gamma e^{-\alpha t} X$. In this case, $\lambda=-\alpha$ and $\|V(t)\|_{\max }=\Gamma e^{-\alpha t}$.

The $L^{1}$-norm defined in Ref. [17] is $\int_{0}^{T}\|H(t)\|_{\max } d t$, which has a linear scaling $\mathcal{O}(h T)$ with the simulation duration $T$, whereas our discretized $L^{1}$-norm $\sum_{w=0}^{r-1}\left\|V\left(t_{w}\right)\right\|_{\max } \Delta t_{w}$ tends to a constant in the long time limit. This can be seen from the fact that the partition terminates at a large enough time $t_{w}(\leq T)$, where $\Delta t_{w}=$ $T-t_{w}$ becomes the final simulation step, as described in Sec. III C 1. The above results also hold for any combination of exponential decays (even when these are multiplied by oscillatory terms) with which different time decay dependencies may be constructed.

\section{E. Hamiltonians with arbitrary time dependence}

The simulation algorithm invokes a switch to the interaction picture, by dividing the Hamiltonian into a static diagonal part $H_{0}$ and a time-dependent Hermitian operator $V(t)$. The $V(t)$ is expanded using permutations and exponential sums as presented in Eq. (16). There, we assume that the time dependence can be expressed as exponential sums with a finite number of terms, $K$. Although this assumption holds for many models (e.g., when the 
TABLE I. A summary of resources for the circuit.

\begin{tabular}{lcc}
\hline \hline Unitary & \multicolumn{1}{c}{ Gate cost } & Qubit cost \\
\hline$e^{-i H_{0} T}$ & $\mathcal{O}(L d)$ & $\mathcal{O}(1)$ \\
$V_{c}$ & $\mathcal{O}\left[Q^{2}+Q M\left(k_{\mathrm{od}}+\log M\right)+Q M K\left(C_{D}+C_{\Delta H_{0}}+C_{\Lambda}\right)\right]$ & $\mathcal{O}(Q \log M K)$ \\
$U_{I}(T)$ & $\mathcal{O}\left\{r\left[Q^{2}+Q M\left(k_{\mathrm{od}}+\log M\right)+Q M K\left(C_{D}+C_{\Delta H_{0}}+C_{\Lambda}\right)\right]\right\}$ & $\mathcal{O}(Q \log M K)$ \\
\hline \hline
\end{tabular}

time dependencies are some combinations of trigonometric functions and exponential decays), the exponential series generally requires an infinite sum (e.g., a Fourier series). A straightforward procedure to obtain a finite sum approximation is via a truncated Fourier series. As an example, let us consider a polynomial function of time, i.e., $f(t)=$ $\sum_{l=0}^{p} c_{l} t^{l}$. Using the proof of Theorem 8.14 in Ref. [38], it can be shown that a truncated Fourier series of $f(t)$ is $\mathcal{O}(\epsilon)$ close to $f(t)$ when the truncation order is $\mathcal{O}(1 / \epsilon)$. We also note that, other than the Fourier series, there have been numerous studies [27-31] regarding finding an exponential-sum approximation of a function. Some of them, e.g., Ref. [27], provide efficient algorithms with logarithmically scaling terms (with respect to the inverse of a required accuracy). These results suggest that efficient methods for finding the exponential-sum decompositions of the time dependencies of $V(t)$ can exist in many cases.

Suppose that $V(t)$ is approximated by a finite series of an exponential sum. The resulting error of the unitary evolution, due to the Hamiltonian approximation, scales only at most linearly with the evolution duration. This can be shown using the following property. Given two time-dependent Hamiltonians $H_{1}(t)$ and $H_{2}(t)$ such that

$$
\left\|H_{1}(t)-H_{2}(t)\right\| \leq \epsilon \quad \text { for all } t \in[0, T],
$$

then

$$
\begin{aligned}
& \left\|U_{1}(T, 0)-U_{2}(T, 0)\right\| \equiv \| \mathcal{T} \exp \left[-i \int_{0}^{T} H_{1}(t) d t\right] \\
& \quad-\mathcal{T} \exp \left[-i \int_{0}^{T} H_{2}(t) d t\right] \| \\
& \leq \epsilon T .
\end{aligned}
$$

This holds for any norm $\|\cdot\|$. Before proving this, we first note a property of the so-called subadditivity of error in implementing unitaries [33]. It says that, for unitaries $U_{1}, U_{2}, V_{3}$, and $V_{4}$, we have

$$
\left\|U_{2} U_{1}-V_{2} V_{1}\right\| \leq\left\|U_{2}-V_{2}\right\|+\left\|U_{1}-V_{1}\right\| .
$$

This can be easily shown by

$$
\begin{aligned}
\left\|U_{2} U_{1}-V_{2} V_{1}\right\| & =\left\|U_{2} U_{1}-V_{2} U_{1}+V_{2} U_{1}-V_{2} V_{1}\right\| \\
& \leq\left\|\left(U_{2}-V_{2}\right) U_{1}\right\|+\left\|V_{2}\left(U_{1}-V_{1}\right)\right\| \\
& \leq\left\|U_{2}-V_{2}\right\|+\left\|U_{1}-V_{1}\right\|
\end{aligned}
$$

where the basic operator norm inequalities are used. Now we prove the bound in Eq. (63). We divide $T$ into $n$ segments such that each segment has width $T / n$. We can rewrite the time evolution operators as

$$
\begin{aligned}
& U_{1}(T, 0)=U_{1}\left(T, \frac{n-1}{n} T\right) \cdots U_{1}\left(\frac{T}{n}, 0\right), \\
& U_{2}(T, 0)=U_{2}\left(T, \frac{n-1}{n} T\right) \cdots U_{2}\left(\frac{T}{n}, 0\right) .
\end{aligned}
$$

Repeatedly using the subadditivity of error, we have

$$
\begin{aligned}
& \left\|U_{1}(T, 0)-U_{2}(T, 0)\right\| \\
& \leq \sum_{m=1}^{n}\left\|U_{1}\left(\frac{m T}{n}, \frac{(m-1) T}{n}\right)-U_{2}\left(\frac{m T}{n}, \frac{(m-1) T}{n}\right)\right\| \\
& \leq \sum_{m=1}^{n}\left\|\int_{(m-1) T / n}^{m T / n}\left[H_{1}(t)-H_{2}(t)\right] d t\right\|+\sum_{m=1}^{n} \mathcal{O}\left[\left(\frac{T}{n}\right)^{2}\right] \\
& \leq \sum_{m=1}^{n} \epsilon \frac{T}{n}+\sum_{m=1}^{n} \mathcal{O}\left[\left(\frac{T}{n}\right)^{2}\right] \\
& =\epsilon T+\mathcal{O}\left[\frac{T^{2}}{n}\right]
\end{aligned}
$$

TABLE II. A comparison of gate costs with the best existing algorithms. Both approaches in Ref. [17] have $H(t)=\sum_{\ell=1}^{L^{\prime}} \alpha_{\ell}(t) H_{\ell}$, where the $H_{\ell}$ are some unitary operators and $\alpha(t)$ some time-dependent coefficients. Here $\|\alpha\|_{1,1} \equiv \sum_{\ell=1}^{L^{\prime}} \int_{0}^{T} d t \alpha_{\ell}(t)\left\|H_{\ell}\right\|_{\infty},\|\alpha\|_{\infty, 1} \equiv$ $\int_{0}^{T} d t|| \alpha_{\ell}(t) \|_{\infty}, g_{c}$ is the gate cost for implementing each controlled $H_{\ell}$, and $g_{e}$ is the gate cost for each controlled $e^{-i T H_{\ell}}$.

\begin{tabular}{lc}
\hline \hline Algorithms & Gate complexity \\
\hline Continuous qDRIFT in Ref. [17] & $\mathcal{O}\left(\|\alpha\|_{1,1}^{2} g_{e} / \epsilon\right)$ \\
Rescaled Dyson series in Ref. [17] & $\tilde{\mathcal{O}}\left(\|\alpha\|_{\infty, 1} L^{\prime 2} g_{c}\right)$ \\
This paper & $\mathcal{O}\left\{r\left[Q^{2}+Q M\left(k_{\text {od }}+\log M\right)+Q M K\left(C_{D}+C_{\Delta H_{0}}+C_{\Lambda}\right)\right]+L d\right\}$ \\
\hline \hline
\end{tabular}


Since this inequality holds for any $n$, we can take $n \rightarrow \infty$ and it yields Eq. (63) as claimed.

Now we apply this property to the simulation of $U_{I}(T)$. Suppose that we have a $\tilde{\delta}$-accurate approximation of $V(t)$, i.e., $\|\tilde{V}(t)-V(t)\| \leq \tilde{\delta}$ for all $t \in[0, T]$, where $\tilde{V}(t)$ is the finite exponential-sum approximation of $V(t)$. The accumulative error from this approximation is bounded by $\tilde{\delta} T$ and the overall error is $\mathcal{O}(\tilde{\delta} T+\delta)$, where $\delta$ is the error from the LCU implementation. Recall that $\tilde{\delta}$ is closely related to $K$ (the number of terms). Although it is intuitive that a larger $K$ can allow for a smaller $\tilde{\delta}$, the explicit relation between the two largely depends on the model and the expansion method. Nonetheless, we can expect $K$ to scale at least linearly with $1 / \tilde{\delta}$ for many cases, e.g., the aforementioned truncated Fourier series for a polynomial.

The simulation cost also depends on $M$, the number of terms in the permutation expansion. This quantity usually scales linearly with the system size and can be easily determined. For example, a typical spin model usually involves a sum of tensor products of Pauli- $X$ (or $Y$ ) operators and Pauli- $Z$. Each tensor product represents an interaction between qubits on certain lattice sites. Because of the common locality constraint that prevents a qubit interacting with those arbitrarily far apart, the number of interacting terms, $M$, scales at most linearly with the number of qubits. In addition, a tensor product of Pauli operators can be easily separated into a product of diagonal matrices and a permutation, e.g., $X \otimes X \otimes Y=(I \otimes I \otimes-i Z)(X \otimes X \otimes$ $X)$. We conclude that $M$ will have modest linear scaling for most practical models.

\section{ALTERNATIVE SCHEME AND REDUCTION TO THE TIME-INDEPENDENT CASE}

In this section, we provide an alternative yet equivalent scheme for the dynamical simulation, one that will allow us to establish an immediate connection to the timeindependent Hamiltonian simulation formalism (specifically to the scheme presented in Ref. [34]), in which $H(t)$ is assumed constant in time.

In previous sections, we have chosen to partition the interaction-picture unitary $U_{I}(T)$ into short time segments and then follow its execution by the application of a diagonal $e^{i H_{0} T}$, bringing it back to the Schrödinger picture. Here, we show that the Schrödinger picture $U(T)$ can be partitioned similarly.

Recalling the expansion of $U_{I}\left(t_{w}+\Delta t_{w}, t_{w}\right)$ in Eq. (31), we have

$$
\begin{aligned}
U_{I}\left(t_{w}\right. & \left.+\Delta t_{w}, t_{w}\right)|z\rangle \\
& =\sum_{q=0}^{\infty} \sum_{\mathbf{i}_{q}} \sum_{\mathbf{k}_{q}}(-i)^{q} e^{t_{w} x_{1}} e^{\Delta t_{w}\left[x_{1}, x_{2}, \ldots, x_{q}, 0\right]} d_{\mathbf{i}_{q}, z}^{\left(\mathbf{k}_{q}\right)} P_{\mathbf{i}_{q}}|z\rangle
\end{aligned}
$$

with

$$
x_{1}=i\left(E_{z_{\mathrm{i}_{q}}}-E_{z}\right)+\sum_{l=1}^{q} \lambda_{i_{l, z_{\mathrm{i}}}}^{\left(k_{l}\right)}
$$

Breaking the $e^{t_{w} x_{1}}$ phase, we get

$$
\begin{aligned}
& U_{I}\left(t_{w}+\Delta t_{w}, t_{w}\right)|z\rangle \\
& \quad=\sum_{q=0}^{\infty} \sum_{\mathbf{i}_{q}} \sum_{\mathbf{k}_{q}}(-i)^{q} e^{t_{w} \sum_{l=1}^{q} \lambda_{i l, \mathbf{i}_{\mathbf{i}}}} e^{-i t_{w} E_{z}} e^{i\left(t_{w}+\Delta t_{w}\right) E_{\mathbf{i}_{q}}} \\
& \times e^{-i \Delta t_{w} E_{\mathbf{z}_{\mathbf{i}}}} e^{\Delta t_{w}\left[x_{1}, x_{2}, \ldots, x_{q}, 0\right]} d_{\mathbf{i}_{q}, z}^{\left(\mathbf{k}_{q}\right)} P_{\mathbf{i}_{q}}|z\rangle
\end{aligned}
$$

We find that

$$
e^{-i H_{0}\left(t_{w}+\Delta t_{w}\right)} U_{I}\left(t_{w}+\Delta t_{w}, t_{w}\right)=\widetilde{U}_{I}\left(t_{w}+\Delta t_{w}, t_{w}\right) e^{-i H_{0} t_{w}},
$$

where

$$
\begin{aligned}
& \widetilde{U}_{I}\left(t_{w}+\Delta t_{w}, t_{w}\right) \\
& =\sum_{z} \sum_{q=0}^{\infty} \sum_{\mathbf{i}_{q}} \sum_{\mathbf{k}_{q}}(-i)^{q} e^{t_{w} \sum_{l=1}^{q} \lambda_{i_{l}, \mathbf{i}_{l}}^{\left(k_{l}\right)}} \\
& \quad \times e^{-i \Delta t_{w} E_{z_{\mathbf{i}_{q}}}} e^{\Delta t_{w}\left[x_{1}, x_{2}, \ldots, x_{q}, 0\right]} d_{\mathbf{i}_{q}, z}^{\left(\mathbf{k}_{q}\right)} P_{\mathbf{i}_{q}}|z\rangle\langle z| .
\end{aligned}
$$

Inspecting the full unitary evolution, we observe that

$$
\begin{aligned}
U(T) & =e^{-i H_{0} T} U_{I}(T) \\
& =e^{-i H_{0} T} U_{I}\left(T, t_{r-1}\right) U_{I}\left(t_{r-1}, t_{r-2}\right) \cdots U_{I}\left(t_{1}, 0\right) \\
& =\widetilde{U}_{I}\left(T, t_{r-1}\right) D\left(t_{r-1}\right) U_{I}\left(t_{r-1}, t_{r-2}\right) \cdots U_{I}\left(t_{1}, 0\right) .
\end{aligned}
$$

The evolution operator $U(T)$ can be simplified as

$$
U(T)=\widetilde{U}_{I}\left(T, t_{r-1}\right) \widetilde{U}_{I}\left(t_{r-1}, t_{r-2}\right) \cdots \widetilde{U}_{I}\left(t_{1}, 0\right),
$$

eliminating the diagonal piece. Each $\widetilde{U}_{I}\left(t_{w}+\Delta t_{w}, t_{w}\right)$ can be rewritten as

$$
\begin{aligned}
& \widetilde{U}_{I}\left(t_{w}+\Delta t_{w}, t_{w}\right) \\
& =\sum_{z} \sum_{q=0}^{\infty} \sum_{\mathbf{i}_{q}} \sum_{\mathbf{k}_{q}}(-i)^{q} e^{\left(t_{w}+\Delta t_{w}\right) \sum_{l=1}^{q} \lambda_{i_{l}, \mathbf{i}_{l}}^{\left(k_{l}\right)}} \\
& \quad \times e^{-\Delta t_{w}\left(i E_{\mathbf{z}_{q}}+\sum_{l=1}^{q} \lambda_{i_{l}, \mathbf{i}_{i} l}^{\left(k_{l}\right)}\right)} e^{\Delta t_{w}\left[x_{1}, x_{2}, \ldots, x_{q}, 0\right]} d_{\mathbf{i}_{q}, z}^{\left(\mathbf{k}_{q}\right)} P_{\mathbf{i}_{q}}|z\rangle\langle z| .
\end{aligned}
$$


The factor $e^{-\Delta t_{w}\left(i E_{\mathbf{z}_{q}}+\sum_{l=1}^{q} \lambda_{i_{l}, \mathbf{z}_{l}}^{\left(k_{l}\right)}\right)}$ can be absorbed into the divided difference

$$
\begin{aligned}
\widetilde{U}_{I}\left(t_{w}+\Delta t_{w}, t_{w}\right)= & \sum_{z} \sum_{q=0}^{\infty} \sum_{\mathbf{i}_{q}} \sum_{\mathbf{k}_{q}}(-i)^{q} e^{\left(t_{w}+\Delta t_{w}\right) \sum_{l=1}^{q} \lambda_{i_{l}, \mathbf{i}_{l}}^{\left(k_{l}\right)}} \\
& \times e^{\Delta t_{w}\left[\tilde{y}_{1}, \tilde{y}_{2}, \ldots, \tilde{y}_{q}, \tilde{y}_{q+1}\right]} d_{\mathbf{i}_{q}, z}^{\left(\mathbf{k}_{q}\right)} P_{\mathbf{i}_{q}}|z\rangle\langle z|
\end{aligned}
$$

with

$$
\begin{aligned}
& \tilde{y}_{j}=x_{j}-\left(i E_{z_{\mathbf{i}_{q}}}+\sum_{l=1}^{q} \lambda_{i_{l}, z_{\mathbf{i}_{l}}}^{\left(k_{l}\right)}\right) \\
& =i\left(E_{z_{\mathbf{i}_{q}}}-E_{z_{\mathbf{i}_{j-1}}}\right)+\sum_{l=j}^{q} \lambda_{i_{l}, \mathbf{z}_{\mathbf{i}_{l}}}^{\left(k_{l}\right)}-\left(i E_{z_{\mathbf{i}_{q}}}+\sum_{l=1}^{q} \lambda_{i_{l}, \mathbf{z}_{l}}^{\left(k_{l}\right)}\right),
\end{aligned}
$$

which simplifies to

$$
\tilde{y}_{j}=-i E_{z_{\mathbf{i}_{j-1}}}-\sum_{l=1}^{j-1} \lambda_{i_{l}, \mathbf{z}_{\mathbf{i}_{l}}}^{\left(k_{l}\right)} .
$$

By inserting additional $i \Delta t_{w} E_{z}$ phases into the divided differences, we can rewrite

$$
\begin{aligned}
\widetilde{U}_{I}\left(t_{w}\right. & \left.+\Delta t_{w}, t_{w}\right) \\
= & \left(\sum_{z} \sum_{q=0}^{\infty} \sum_{\mathbf{i}_{q}} \sum_{\mathbf{k}_{q}}(-i)^{q} e^{\left(t_{w}+\Delta t_{w}\right) \sum_{l=1}^{q} \lambda_{i_{l}, \mathbf{i}_{i}}^{\left(k_{l}\right)}}\right. \\
\times & \left.e^{\Delta t_{w}\left[y_{1}, y_{2}, \ldots, y_{q}, y_{q+1}\right]} d_{\mathbf{i}_{q}, z}^{\left(\mathbf{k}_{q}\right)} P_{\mathbf{i}_{q}}|z\rangle\langle z|\right) \\
& \times e^{-i H_{0} \Delta t_{w}}
\end{aligned}
$$

with

$$
\begin{aligned}
y_{j}=\tilde{y}_{j}+i E_{z} & =-i\left(E_{z_{\mathbf{i}_{j-1}}}-E_{z}\right)-\sum_{l=1}^{j-1} \lambda_{i_{l}, \mathbf{z}_{l}}^{\left(k_{l}\right)} \\
& =-i \Delta E_{z_{\mathbf{i}_{j-1}}}-\sum_{l=1}^{j-1} \lambda_{i_{l}, \mathbf{z}_{l}}^{\left(k_{l}\right)} .
\end{aligned}
$$

Now, we can write $U(T)$ as alternating off-diagonal and diagonal unitaries:

$$
\begin{aligned}
U(T) & =\prod_{w} \widetilde{U}_{I}\left(t_{w}+\Delta t_{w}, t_{w}\right) \\
& \equiv \prod_{w} U_{\text {od }}\left(t_{w}+\Delta t_{w}, t_{w}\right) e^{-i H_{0} \Delta t_{w}} .
\end{aligned}
$$

When $H(t)$ becomes time independent, $\lambda_{i_{l}, z_{i_{l}}}^{\left(k_{l}\right)}=0$ and $\Delta t_{w}=\Delta t=\ln 2 / \Gamma$. To synchronize the notation with that of Ref. [34], we identify $H_{0}=D_{0}$ and $U_{\text {od }}\left(t_{w}+\right.$ $\left.\Delta t_{w}, t_{w}\right)=U_{\text {od }}$. The evolution operator becomes $U(T)=$ $U_{\text {od }} e^{-i D_{0} \Delta t} \cdots U_{\text {od }} e^{-i D_{0} \Delta t}$, which coincides with Ref. [34].

\section{CONCLUSIONS}

We have presented a quantum algorithm for simulating the evolution operator generated from a time-dependent Hamiltonian. The algorithm involves a permutation expansion for the interaction Hamiltonian, a switch to the interaction picture, and the incorporation of the LCU technique. Combining the permutation expansion with the Dyson series leads to an integral-free representation for the interaction-picture unitary with coefficients involving the notion of divided differences with complex inputs.

We find that our expansion allows us to adjust the time steps based on the dynamical characteristics of the Hamiltonian, providing a resource saving as compared to the equal-size partition with the largest bound. This further results in a gate resource that scales with an $L^{1}$-normlike scaling with respect only to the "nonstatic" norm of the Hamiltonian. Our expansion also improves on the traditional product-formula algorithms, where the evolution operator is divided into equal-size pieces, where each piece is approximated by a time-independent Hamiltonian simulation, i.e., $e^{-i H_{t} d t}$.

Specifically, we have demonstrated that, for systems with a decaying nonstatic component, the resources do not scale with the total evolution time asymptotically. Furthermore, the simulation cost is independent of the frequencies, implying a significant advantage for systems with highly oscillating components.

\section{ACKNOWLEDGMENTS}

This work is supported by the U.S. Department of Energy (DOE), Office of Science, Basic Energy Sciences (BES) under Award No. DE-SC0020280.

\section{APPENDIX A: PROPERTIES OF DIVIDED DIFFERENCE}

We begin with a formal definition of divided difference for complex-valued functions and follow with some properties that will be of use to us when deriving the new bound. The main results are derived for the exponential functions.

Definition 1. Let $\mathbb{U}$ be an open subset of $\mathbb{C}$, and $f$ : $\mathbb{U} \rightarrow \mathbb{C}$ is analytic in $\mathbb{U}$. For any non-negative integer $q$ and $x_{0}, x_{1}, \ldots, x_{q} \in \mathbb{U}$, the divided difference of $f$ is denoted as $f\left[x_{0}, x_{1}, \ldots, x_{q}\right]$. If $q=0, f\left[x_{0}\right] \equiv f\left(x_{0}\right)$. Suppose that $\left\{x_{0}, x_{1}, \ldots, x_{q}\right\}$ has $r$ distinct elements. Let $S=$ $\left\{x_{\sigma(0)}, x_{\sigma(1)}, \ldots, x_{\sigma(q)}\right\}$ be a sorted set of $\left\{x_{0}, x_{1}, \ldots, x_{q}\right\}$, i.e., there exists a permutation $\sigma$ such that the first $n_{1}$ elements of $S$ are equal and the following $n_{2}$ elements of $S$ are equal, and so on and so forth. There are r same-element clusters and $\sum_{i=1}^{r} n_{i}=q+1$. The divided difference of $f$ 
is defined as

$$
\begin{aligned}
f & {\left[x_{0}, x_{1}, \ldots, x_{q}\right] } \\
& = \begin{cases}\frac{f\left[x_{\sigma(1)}, \ldots, x_{\sigma(q)}\right]-f\left[x_{\sigma(0)}, \ldots, x_{\sigma(q-1)}\right]}{x_{\sigma(q)}-x_{\sigma(0)}} & \text { if } r>1, \\
\frac{f_{(q)}\left(x_{0}\right)}{q !} & \text { if } r=1,\end{cases}
\end{aligned}
$$

where $f^{(q)}$ denotes the $q$ th derivative of $f$.

Although the above sorting procedure is not unique, it can be shown that any choice of the permutation gives the same result, and hence the definition is well defined.

The divided difference involves a recursive relation that connects a $q+1$ input case to two $q$ cases. For $q=1$,

$$
f\left[x_{0}, x_{1}\right]= \begin{cases}\frac{f\left(x_{1}\right)-f\left(x_{0}\right)}{x_{1}-x_{0}} & \text { if } x_{0} \neq x_{1}, \\ f^{\prime}\left(x_{0}\right) & \text { if } x_{0}=x_{1} .\end{cases}
$$

For $q=2$, and suppose $x_{0}, x_{1}$ and $x_{2}$ are all distinct,

$$
\begin{aligned}
f\left[x_{0}, x_{1}, x_{2}\right]= & \frac{1}{x_{2}-x_{0}}\left(\frac{f\left(x_{2}\right)-f\left(x_{1}\right)}{x_{2}-x_{1}}-\frac{f\left(x_{1}\right)-f\left(x_{0}\right)}{x_{1}-x_{0}}\right) \\
= & \frac{f\left(x_{0}\right)}{\left(x_{0}-x_{1}\right)\left(x_{0}-x_{2}\right)}+\frac{f\left(x_{1}\right)}{\left(x_{1}-x_{2}\right)\left(x_{1}-x_{0}\right)} \\
& +\frac{f\left(x_{2}\right)}{\left(x_{2}-x_{0}\right)\left(x_{2}-x_{1}\right)} .
\end{aligned}
$$

In fact, it can be shown that, for distinct $x_{0}, x_{1}, \ldots, x_{q}$,

$$
f\left[x_{0}, x_{1}, \ldots, x_{q}\right]=\sum_{i=0}^{q} \frac{f\left(x_{i}\right)}{\prod_{k \neq i}\left(x_{i}-x_{k}\right)} .
$$

Remark. Since any analytic function admits a Taylor expansion representation and the divided difference is a linear functional, the divided difference of an analytic function $f$ has a series expansion form, i.e., for $x_{0}, \ldots, x_{q}$ and $y$ in $f$ 's analytic domain,

$$
f\left[x_{0}, \ldots, x_{q}\right]=\sum_{n=0}^{\infty} \frac{f^{(n)}(y)}{n !} p_{n \mid y}\left[x_{0}, \ldots, x_{q}\right],
$$

where $p_{n \mid y}(x) \equiv(x-y)^{n}$. Because $p_{n \mid y}\left[x_{0}, \ldots, x_{q}\right]=0$ for all $n<q$, the nonvanishing term of the series starts from the $q$ th order.

For simplicity, we denote the divided difference for the exponential function as $e^{\left[x_{0}, \ldots, x_{q}\right]}$, i.e.,

$$
e^{\left[x_{0}, \ldots, x_{q}\right]} \equiv f\left[x_{0}, \ldots, x_{q}\right], \quad \text { where } f(x)=e^{x} .
$$

Property 1. For any non-negative integer $q$ and $x_{0}, x_{1}, \ldots, x_{q} \in \mathbb{C}$,

$$
e^{\left[x_{0}, x_{1}, \ldots, x_{q}\right]}=e^{x_{0}} e^{\left[0, x_{1}-x_{0}, \ldots, x_{q}-x_{0}\right]} .
$$

This property and the fact that divided differences are permutation symmetric among inputs imply that any input can be factored out of the divided difference by subtracting it from every entry.

Property 2. For any non-negative integer $q$ and $x_{0}, x_{1}, \ldots, x_{q} \in \mathbb{C}$,

$$
e^{\left[x_{0}, x_{1}, \ldots, x_{q}\right]}=\sum_{n=q}^{\infty} \frac{1}{n !} \sum_{\sum k_{j}=n-q} \prod_{j=0}^{q}\left(x_{j}\right)^{k_{j}} .
$$

An equivalent definition of the divided difference for an analytic function is via its Taylor expansion. It amounts to applying the divided difference on every order of the series. Since any polynomial of order less than $q$ is annihilated, the series starts from the order $q$. Property 2 is derived from the Taylor expansion of $e^{x}$ with respect to the origin.

Lemma 1. For any non-negative integer $q$ and $x_{0}, x_{1}, \ldots$, $x_{q} \in \mathbb{C}$,

$$
\int_{0}^{1} a^{q} e^{\left[a x_{0}, a x_{1}, \ldots, a x_{q}\right]} d a=e^{\left[0, x_{0}, x_{1}, \ldots, x_{q}\right]}
$$

Proof. This can be observed from the series expansion of the divided difference for the exponential function, i.e., from Property 2,

$$
\begin{aligned}
a^{q} e^{\left[a x_{0}, a x_{1}, \ldots, a x_{q}\right]} & =a^{q} \sum_{n=q}^{\infty} \frac{1}{n !} \sum_{\sum k_{j}=n-q j=0} \prod^{q}\left(a x_{j}\right)^{k_{j}} \\
& =a^{q} \sum_{n=q}^{\infty} \frac{1}{n !} \sum_{\sum k_{j}=n-q} a^{n-q} \prod_{j=0}^{q}\left(x_{j}\right)^{k_{j}} \\
& =\sum_{n=q}^{\infty} \frac{a^{n}}{n !} \sum_{\sum k_{j}=n-q j=0} \prod_{j}^{q}\left(x_{j}\right)^{k_{j}} .
\end{aligned}
$$

Performing term-by-term integration over $a$ on both sides, we have

$$
\begin{aligned}
& \int_{0}^{1} a^{q} e^{\left[a x_{0}, a x_{1}, \ldots, a x_{q}\right]} d a \\
& =\sum_{n=q}^{\infty}\left(\int_{0}^{1} \frac{a^{n}}{n !} d a\right) \sum_{\sum k_{j}=n-q} \prod_{j=0}^{q}\left(x_{j}\right)^{k_{j}} \\
& =\sum_{n=q}^{\infty} \frac{1}{(n+1) !} \sum_{\sum k_{j}=n-q} \prod_{j=0}^{q}\left(x_{j}\right)^{k_{j}} \\
& =e^{\left[0, x_{0}, x_{1}, \ldots, x_{q}\right]} \text {, }
\end{aligned}
$$

where the last equality follows from the series expansion representation of $e^{\left[0, x_{0}, x_{1}, \ldots, x_{q}\right]}$. This completes the proof. 
Corrollary 1. Let $f(x)=e^{t x}$, where $t \in \mathbb{R}$ and $x \in \mathbb{C}$. We let $e^{t\left[x_{0}, \ldots, x_{q}\right]} \equiv f\left[x_{0}, \ldots, x_{q}\right]$, where $x_{0}, \ldots, x_{q} \in \mathbb{C}$. For any $\tau \in \mathbb{R}$,

$$
\int_{0}^{\tau} e^{t\left[x_{0}, \ldots, x_{q}\right]} d t=e^{\tau\left[0, x_{0}, \ldots, x_{q}\right]} .
$$

This can be verified by evaluating the series expansion form on both sides, by a similar manner as in the proof of Lemma 1.

With these properties, we are ready to prove the bound in Identity 2 in the main text.

Theorem 1. For any non-negative integer $q$ and $x_{0}, x_{1}, \ldots, x_{q} \in \mathbb{C}$,

$$
\left|e^{\left[x_{0}, x_{1}, \ldots, x_{q}\right]}\right| \leq e^{\left[\operatorname{Re}\left(x_{0}\right), \operatorname{Re}\left(x_{1}\right), \ldots, \operatorname{Re}\left(x_{q}\right)\right]},
$$

where $\operatorname{Re}(\cdot)$ gives the real part of the input.

Proof. We proceed by induction. Equation (A13) is trivially satisfied with the equality when $q=0$. For the case $q=1$, we have

$$
\begin{aligned}
\left|e^{\left[x_{0}, x_{1}\right]}\right| & =\left|e^{x_{0}}\right|\left|e^{\left[0, x_{1}-x_{0}\right]}\right| \\
& =e^{\operatorname{Re}\left(x_{0}\right)}\left|\int_{0}^{1} a e^{a\left(x_{1}-x_{0}\right)} d a\right| \\
& \leq e^{\operatorname{Re}\left(x_{0}\right)} \int_{0}^{1} a\left|e^{a\left(x_{1}-x_{0}\right)}\right| d a \\
& =e^{\operatorname{Re}\left(x_{0}\right)} \int_{0}^{1} a e^{a \operatorname{Re}\left(x_{1}-x_{0}\right)} d a \\
& =e^{\left[\operatorname{Re}\left(x_{0}\right), \operatorname{Re}\left(x_{1}\right)\right]},
\end{aligned}
$$

where Lemma 1 has been used. Assume that we have

$$
\left|e^{\left[x_{0}, \ldots, x_{q}\right]}\right| \leq e^{\left[\operatorname{Re}\left(x_{0}\right), \ldots, \operatorname{Re}\left(x_{q}\right)\right]},
$$

which is true for $q=0,1$. It follows that

$$
\begin{aligned}
& \left|e^{\left[x_{0}, \ldots, x_{q}, x_{q+1}\right]}\right| \\
& \quad=\left|e^{x_{q+1}}\right|\left|e^{\left[0, x_{0}-x_{q+1}, \ldots, x_{q}-x_{q+1}\right]}\right| \\
& \quad=e^{\operatorname{Re}\left(x_{q+1}\right)}\left|\int_{0}^{1} a^{q} e^{\left[a\left(x_{0}-x_{q+1}\right), \ldots, a\left(x_{q}-x_{q+1}\right)\right]} d a\right| \\
& \quad \leq e^{\operatorname{Re}\left(x_{q+1}\right)} \int_{0}^{1} a^{q}\left|e^{\left[a\left(x_{0}-x_{q+1}\right), \ldots, a\left(x_{q}-x_{q+1}\right)\right]}\right| d a \\
& \quad \leq e^{\operatorname{Re}\left(x_{q+1}\right)} \int_{0}^{1} a^{q} e^{\left[\operatorname{Re}\left(a x_{0}-a x_{q+1}\right), \ldots, \operatorname{Re}\left(a x_{q}-a x_{q+1}\right)\right]} d a \\
& \quad=e^{\operatorname{Re}\left(x_{q+1}\right)} e^{\left[0, \operatorname{Re}\left(x_{0}-x_{q+1}\right), \ldots, \operatorname{Re}\left(x_{q}-x_{q+1}\right)\right]} \\
& \quad=e^{\left[\operatorname{Re}\left(x_{0}\right), \ldots, \operatorname{Re}\left(x_{q}\right), \operatorname{Re}\left(x_{q+1}\right)\right]},
\end{aligned}
$$

where the second and third equalities use Lemma 1 and the second inequality uses Eq. (A15). This proves that the inequality holds for any number of complex inputs.

\section{APPENDIX B: BOUNDING $\left|e^{\Delta t_{w}\left[x_{1}, x_{2}, \ldots, x_{q}, 0\right]}\right|$}

For $\left|e^{\Delta t_{w}\left[x_{1}, x_{2}, \ldots, x_{q}, 0\right]}\right|$, we use the following theorem.

Theorem 2. For any $q+1$ complex values $x_{0}, \ldots, x_{q} \in \mathbb{C}$,

$$
\left|e^{\left[x_{0}, \ldots, x_{q}\right]}\right| \leq e^{\left[\operatorname{Re}\left(x_{0}\right), \ldots, \operatorname{Re}\left(x_{q}\right)\right]},
$$

where $\operatorname{Re}(\cdot)$ gives the real part of the input.

This is proved in Appendix A. From this, we have

$$
\begin{aligned}
\left|e^{\Delta t_{w}\left[x_{1}, \ldots, x_{q}, 0\right]}\right| & =\left(\Delta t_{w}\right)^{q}\left|e^{\left[\Delta t_{w} x_{1}, \ldots, \Delta t_{w} x_{q}, 0\right]}\right| \\
& \leq\left(\Delta t_{w}\right)^{q} e^{\left[\Delta t_{w} \operatorname{Re}\left(x_{1}\right), \ldots, \Delta t_{w} \operatorname{Re}\left(x_{q}\right), 0\right]} .
\end{aligned}
$$

From the definition of $x_{j}$, we have, for all $j \in\{1, \ldots, q\}$,

$$
\operatorname{Re}\left(x_{j}\right)=\sum_{l=j}^{q} \operatorname{Re}\left(\lambda_{i_{l}, z_{\mathbf{i}_{l}}}^{\left(k_{l}\right)}\right) \leq(q-j+1) \lambda .
$$

Based on the property that increasing any input in $e^{[,, \ldots, \cdot]}$ will only increase its value (which can be proved by taking derivatives in the Hermite-Genocchi form), we have

$$
\begin{aligned}
\left|e^{\Delta t_{w}\left[x_{1}, \ldots, x_{q}, 0\right]}\right| & \leq\left(\Delta t_{w}\right)^{q} e^{\left[\Delta t_{w} \operatorname{Re}\left(x_{1}\right), \ldots, \Delta t_{w} \operatorname{Re}\left(x_{q}\right), 0\right]} \\
& \leq\left(\Delta t_{w}\right)^{q} e^{\left[\Delta t_{w} q \lambda, \Delta t_{w}(q-1) \lambda, \ldots, \Delta t_{w} \lambda, 0\right]} .
\end{aligned}
$$

Using the permutation symmetric property and Property 1 , we have

$$
\begin{aligned}
\left(\Delta t_{w}\right)^{q} e^{\left[\Delta t_{w} q \lambda, \Delta t_{w}(q-1) \lambda, \ldots, \Delta t_{w} \lambda, 0\right]} \\
=\Delta t_{w}{ }^{q} \frac{e^{\left[\Delta t_{w} q \lambda, \Delta t_{w}(q-1) \lambda, \ldots, \Delta t_{w} \lambda\right]}-e^{\left[\Delta t_{w}(q-1) \lambda, \ldots, \Delta t_{w} \lambda, 0\right]}}{\Delta t_{w} \lambda q} \\
=\left(\Delta t_{w}\right)^{q} \frac{e^{\Delta t_{w} \lambda}-1}{\Delta t_{w} \lambda q} e^{\left[\Delta t_{w}(q-1) \lambda, \ldots, \Delta t_{w} \lambda, 0\right]} \\
=\cdots \\
=\left(\frac{e^{\lambda \Delta t_{w}}-1}{\lambda}\right)^{q} \frac{1}{q !} .
\end{aligned}
$$

Therefore, we have

$$
\left|e^{\Delta t_{w}\left[x_{1}, x_{2}, \ldots, x_{q}, 0\right]}\right| \leq \frac{1}{q !}\left(\frac{e^{\lambda \Delta t_{w}}-1}{\lambda}\right)^{q} .
$$




\section{APPENDIX C: LCU METHOD REVIEW}

We give a brief introduction to the LCU method in this section, and we adapt the notation of the original paper [14] for a more convenient reference to readers. Suppose that we have a unitary $U$, which is an infinite sum of unitaries, i.e.,

$$
U=\sum_{j=0}^{\infty} \beta_{j} V_{j}
$$

where $\beta_{j}>0$ and the $V_{j}$ are some unitaries. A truncated series, up to order $m-1$, yields an operator

$$
\tilde{U}=\sum_{j=0}^{m-1} \beta_{j} V_{j}
$$

which approaches $U$ as $m$ increases. We perform the following procedure to effectively implement $\tilde{U}$ on a state $|\psi\rangle$ embedded in a larger system. Prepare an $m$-dimensional ancilla $|0\rangle$ and implement a unitary $B$ such that

$$
B|0\rangle=\frac{1}{\sqrt{s}} \sum_{j=0}^{m-1} \sqrt{\beta_{j}}|j\rangle,
$$

where $s=\sum_{j=0}^{m-1} \beta_{j}$. Suppose that we have access to a control unitary $V_{c}$ such that, for each $j$,

$$
V_{c}|j\rangle|\psi\rangle=|j\rangle V_{j}|\psi\rangle \text {. }
$$

Consider the following combination of the above operations:

$$
W \equiv\left(B^{\dagger} \otimes I\right) V_{c}(B \otimes I) .
$$

We have

$$
W|0\rangle|\psi\rangle=\frac{1}{s}|0\rangle \tilde{U}|\psi\rangle+\sqrt{1-\frac{1}{s^{2}}}|\Phi\rangle,
$$

where $|\Phi\rangle$ 's ancillary part is orthogonal to $|0\rangle\langle 0|$. Let us denote by $P \equiv|0\rangle\langle 0| \otimes I$ the orthogonal projection onto that subspace and by $R \equiv I-2 P$ the reflection operator with respect to $P$. It is shown that the sequence of operations $A \equiv-W R W^{\dagger} R W$ acting on the total system is $A|0\rangle|\psi\rangle=|0\rangle \tilde{U}|\psi\rangle$ when $\tilde{U}$ is unitary and $s=2$. This procedure is the so-called oblivious amplitude amplification. However, $\tilde{U}$ is in general not unitary because it is a truncated series of $U$. This nonunitarity can be accounted for when $\tilde{U} \approx U$ and $s \approx 2$. More specifically, it is shown that if ||$U-\tilde{U}||=\mathcal{O}(\delta)$ and $|s-2|=\mathcal{O}(\delta)$ then

$$
\| P A|0\rangle|\psi\rangle-|0\rangle U|\psi\rangle \|=\mathcal{O}(\delta) .
$$

This means that, when $\tilde{U}$ is $\delta$ close to $U$ and $s$ is $\delta$ close to 2 , the effect of the operator $A$ on the whole system is $\delta$ close to only $U$ acting on $|\psi\rangle$.
Note that the condition $\|U-\tilde{U}\|=\mathcal{O}(\delta)$ can be satisfied when the truncation order $m$ is high enough. However, the condition $|s-2|=\mathcal{O}(\delta)$ is satisfied only when the $\beta_{j}$ are specifically chosen. By construction, we require that $s=\sum_{j=0}^{m-1} \beta_{j}$. If we choose $\beta_{j}=(\ln 2)^{j} / j$ ! then

$$
s=\sum_{j=0}^{m-1} \frac{(\ln 2)^{j}}{j !}
$$

becomes a truncated Taylor expansion of 2, i.e., $2=$ $e^{\ln 2}$. In fact, it can be shown that the required truncation order $m$ such that $|s-2|=\mathcal{O}(\delta)$ scales like $\log (1 / \delta) / \log [\log (1 / \delta)]$. With this $m$, it also guarantees that $\|U-\tilde{U}\|=\mathcal{O}(\delta)$, because

$$
\|U-\tilde{U}\|=\left\|\sum_{j=m}^{\infty} \frac{(\ln 2)^{j}}{j !} V_{j}\right\| \leq \sum_{j=m}^{\infty} \frac{(\ln 2)^{j}}{j !}=|2-s| .
$$

In summary, performing $A$ on an extended system $|0\rangle|\psi\rangle$, with $\beta_{j}=(\ln 2)^{j} / j !$ and $m=\mathcal{O}[\log (1 / \delta) / \log \log (1 / \delta)]$, effectively performs $U$ on $|\psi\rangle$ with $\mathcal{O}(\delta)$ accuracy.

\section{APPENDIX D: AN ALTERNATIVE APPROACH FOR THE LCU SETUP}

We provide an alternative procedure for the LCU routine that leads to an exponential saving for the state preparation. Let us define

$$
\Gamma \equiv \max _{\forall k, i}\left\|D_{i}^{(k)}\right\|_{\max }
$$

Reevaluating the coefficients in Eq. (31) using the $\Gamma$ above, we have

$$
\begin{aligned}
& \left|e^{t_{w} x_{1}} e^{\Delta t_{w}\left[x_{1}, x_{2}, \ldots, x_{q}, 0\right]} d_{\mathbf{i}_{q}, z}^{\left(\mathbf{k}_{q}\right)}\right| \\
& =\frac{\left(\Gamma \widetilde{\Delta t_{w}} e^{t_{w} \lambda}\right)^{q}}{q !} \cos \left[\phi_{\mathbf{i}_{q}, z}^{\left(\mathbf{k}_{q}\right)}\right] e^{i \theta_{\mathbf{i}_{q}, z}^{\left(\mathbf{k}_{q}\right)}} .
\end{aligned}
$$

The evolution operator from $t_{w}$ to $t_{w}+\Delta t_{w}$ becomes

$$
\begin{aligned}
& U_{I}\left(t_{w}+\Delta t_{w}, t_{w}\right) \\
& =\sum_{z} U_{I}\left(t_{w}+\Delta t_{w}, t_{w}\right)|z\rangle\langle z| \\
& =\sum_{z} \sum_{q=0}^{\infty} \sum_{\mathbf{i}_{q}} \sum_{\mathbf{k}_{q}}(-i)^{q} \frac{\left(\Gamma \widetilde{\Delta t_{w}} e^{t_{w} \lambda}\right)^{q}}{2 q !} \\
& \quad \times\left(e^{i \phi_{\mathbf{i}_{q}, z}^{\left(\mathbf{k}_{q}\right)}+i \theta_{\mathbf{i}_{q}, z}^{\left(\mathbf{k}_{q}\right)}}+e^{\left.-i \dot{i}_{\mathbf{i}_{q}, z}^{\left(\mathbf{k}_{q}\right)}+i \theta_{\mathbf{i}_{q}, z}^{\left(\mathbf{k}_{q}\right)}\right) P_{\mathbf{i}_{q}}|z\rangle\langle z|}\right. \\
& =\sum_{q=0}^{\infty} \frac{\left(\Gamma \widetilde{\Delta t_{w}} e^{t_{w} \lambda}\right)^{q}}{2 q !} \sum_{\mathbf{i}_{q}} \sum_{\mathbf{k}_{q}} \sum_{x= \pm}(-i)^{q} P_{\mathbf{i}_{q}} \Phi_{\mathbf{i}_{q}, x}^{\left(\mathbf{k}_{q}, w\right)} .
\end{aligned}
$$


The required state $\left|\psi_{0}\right\rangle$ for $\mathrm{LCU}$ becomes

$$
\left|\psi_{0}\right\rangle=\frac{1}{\sqrt{s}} \sum_{q=0}^{Q} \sqrt{\frac{\left(\Gamma \widetilde{\Delta t_{w}} e^{t_{w} \lambda}\right)^{q}}{2 q !}} \sum_{\mathbf{i}_{q}} \sum_{\mathbf{k}_{q}} \sum_{x=0,1}\left|\mathbf{i}_{q}\right\rangle\left|\mathbf{k}_{q}\right\rangle|x\rangle,
$$

where $s$ is the normalization factor, i.e.,

$$
s=\sum_{q=0}^{Q} \frac{\left(M K \Gamma \widetilde{\Delta t_{w}} e^{t_{w} \lambda}\right)^{q}}{q !} .
$$

To prepare state (D4), we first prepare a state of the following form:

$$
\frac{1}{\sqrt{s}} \sum_{q=0}^{Q} \sqrt{\frac{\left(M K \Gamma \widetilde{\Delta t_{w}} e^{t_{w} \lambda}\right)^{q}}{q !}}|1\rangle^{\otimes q}|0\rangle^{\otimes(Q-q)}|1\rangle^{\otimes q}|0\rangle^{\otimes(Q-q)} .
$$

Subsequently, for each $|1\rangle$ in the first $Q$ registers $\left(\mathbf{i}_{q}\right.$ part), we transform it to $(1 / \sqrt{M}) \sum_{i=0}^{M}|i\rangle$, and for each $|1\rangle$ in the later $Q$ registers ( $\mathbf{k}_{q}$ part), we transform it to $(1 / \sqrt{K}) \sum_{k=1}^{K}|k\rangle$. State (D6) becomes

$$
\begin{aligned}
& \frac{1}{\sqrt{s}} \sum_{q=0}^{Q} \sqrt{\frac{\left(M K \Gamma \widetilde{\Delta t_{w}} e^{t_{w} \lambda}\right)^{q}}{q !}} \sum_{\mathbf{i}_{q}} \frac{1}{\sqrt{M^{q}}}\left|\mathbf{i}_{q}\right\rangle \sum_{\mathbf{k}_{q}} \frac{1}{\sqrt{K^{q}}}\left|\mathbf{k}_{q}\right\rangle \\
& =\frac{1}{\sqrt{s}} \sum_{q=0}^{Q} \sum_{\mathbf{i}_{q}} \sum_{\mathbf{k}_{q}} \sqrt{\frac{\left(\Gamma \widetilde{\Delta t_{w}} e^{t_{w} \lambda}\right)^{q}}{q !}}\left|\mathbf{i}_{q}\right\rangle\left|\mathbf{k}_{q}\right\rangle,
\end{aligned}
$$

which is the required $\left|\psi_{0}\right\rangle$ in Eq. (D4), when combined with $|x\rangle$. Note that, since the transformations $|1\rangle \rightarrow(1 / \sqrt{M}) \sum_{i=0}^{M}|i\rangle$ and $|1\rangle \rightarrow(1 / \sqrt{K}) \sum_{k=1}^{K}|k\rangle$ are mappings to the equally distributed state, they can be done with a column of parallel Hadamard gates, which has a gate $\operatorname{cost} \mathcal{O}[\log (M K)]$. This provides an exponential saving compared to $\mathcal{O}(M K)$ given in the main text. This saving can be apparent when $M K$ becomes large. However, this can create an overhead in the required number of repetitions. Indeed, we have $\Gamma e^{t_{w} \lambda}=M K \max _{\forall k, i}\left\|D_{i}^{(k)}\right\|_{\max } e^{t_{w} \lambda}$ here compared to $\Gamma\left(t_{w}\right)=\sum_{i} \sum_{k}\left\|D_{i}^{(k)}\right\|_{\max } e^{t_{w} \lambda_{(i, k)}}$ in the main text, and the overall simulation cost monotonically increases with this quantity. If only a few $\left\|D_{i}^{(k)}\right\|_{\max } e^{t_{W} \lambda_{(i, k)}}$ are much larger than the others such that $M K \max _{\forall k, i}\left\|D_{i}^{(k)}\right\| \|_{\max } e^{t_{w} \lambda} \gg$ $\sum_{i} \sum_{k}\left\|D_{i}^{(k)}\right\|_{\max } e^{t_{w} \lambda_{(i, k)}}$, then the method provided in the main text is preferred. Depending on the models, one may favor one over the other.

[1] R. P. Feynman, Simulating physics with computers, Int. J. Theor. Phys. 21, 467 (1982).
[2] M. Reiher, N. Wiebe, K. M. Svore, D. Wecker, and M. Troyer, Elucidating reaction mechanisms on quantum computers, Proc. Natl. Acad. Sci. 114, 7555 (2017).

[3] R. Babbush, N. Wiebe, J. McClean, J. McClain, H. Neven, and G. K.-L. Chan, Low-Depth Quantum Simulation of Materials, Phys. Rev. X 8, 011044 (2018).

[4] R. Gioiosa, in Rugged Embedded Systems, edited by A. Vega, P. Bose, and A. Buyuktosunoglu (Morgan Kaufmann, Boston, 2017), p. 123.

[5] T. Sterling, M. Anderson, and M. Brodowicz, in High Performance Computing, edited by T. Sterling, M. Anderson, and M. Brodowicz (Morgan Kaufmann, Boston, 2018), p. 285.

[6] G. Lee, in Cloud Networking, edited by G. Lee (Morgan Kaufmann, Boston, 2014), p. 179.

[7] S. Pang and A. N. Jordan, Optimal adaptive control for quantum metrology with time-dependent Hamiltonians, Nat. Commun. 8, 14695 (2017).

[8] L. J. Butler, CHEMICAL REACTION DYNAMICS BEYOND THE BORN-OPPENHEIMER APPROXIMATION, Annu. Rev. Phys. Chem. 49, 125 (1998), pMID: 15012427.

[9] E. Farhi, J. Goldstone, S. Gutmann, J. Lapan, A. Lundgren, and D. Preda, A quantum adiabatic evolution algorithm applied to random instances of an NP-complete Problem, Science 292, 472 (2001).

[10] E. Farhi, J. Goldstone, and S. Gutmann, arXiv:1411.4028 (2014).

[11] N. Wiebe, D. W. Berry, P. Høyer, and B. C. Sanders, Simulating quantum dynamics on a quantum computer, J. Phys. A: Math. Theor. 44, 445308 (2011).

[12] D. Poulin, A. Qarry, R. Somma, and F. Verstraete, Quantum Simulation of Time-Dependent Hamiltonians and the Convenient Illusion of Hilbert Space, Phys. Rev. Lett. 106, 170501 (2011).

[13] D. W. Berry, A. M. Childs, R. Cleve, R. Kothari, and R. D. Somma, in Proceedings of the Forty-Sixth Annual ACM Symposium on Theory of Computing, STOC '14 (Association for Computing Machinery, New York, NY, USA, 2014), p. 283.

[14] D. W. Berry, A. M. Childs, R. Cleve, R. Kothari, and R. D. Somma, Simulating Hamiltonian Dynamics with a Truncated Taylor Series, Phys. Rev. Lett. 114, 090502 (2015).

[15] G. H. Low and N. Wiebe, 'Hamiltonian simulation in the interaction picture, arXiv:1805.00675 [quant-ph] (2019).

[16] M. Kieferová, A. Scherer, and D. W. Berry, Simulating the dynamics of time-dependent hamiltonians with a truncated dyson series, Phys. Rev. A 99, 042314 (2019).

[17] D. W. Berry, A. M. Childs, Y. Su, X. Wang, and N. Wiebe, Time-dependent Hamiltonian simulation with $L^{1}$ norm scaling, Quantum 4, 254 (2020).

[18] F. J. Dyson, The radiation theories of tomonaga, schwinger, and Feynman, Phys. Rev. 75, 486 (1949).

[19] A. Kalev and I. Hen, An integral-free representation of the dyson series using divided differences, arXiv:2010.09888 [quant-ph] (2020). 
[20] C. de Boor, Divided differences, Surveys Approximation Theory 1, 46 (2005).

[21] P. Davis, Interpolation and Approximation, Dover Books on Mathematics (Dover Publications, New York City, USA, 1976).

[22] A. C. Mccurdy, Ph.D. thesis, University of California, Berkeley 1980, aAI8029490.

[23] L. Gupta, L. Barash, and I. Hen, Calculating the divided differences of the exponential function by addition and removal of inputs, Comput. Phys. Commun. 254, 107385 (2020)

[24] A. McCurdy, K. C. Ng, and B. N. Parlett, Accurate computation of divided differences of the exponential function, Math. Comput. 43, 501 (1984).

[25] F. Zivcovich, Fast and accurate computation of divided differences for analytic functions, with an application to the exponential function, Dolomites Res. Notes Approximation 12, 28 (2019).

[26] L. Gupta, T. Albash, and I. Hen, Permutation matrix representation quantum monte Carlo, J. Stat. Mech.: Theory Exp. 2020, 073105 (2020).

[27] G. Beylkin and L. Monzón, On approximation of functions by exponential sums, Appl. Comput. Harmon. Anal. 19, 17 (2005).

[28] G. Beylkin and L. Monzón, Approximation by exponential sums revisited, Appl. Comput. Harmon. Anal. 28, 131 (2010), special Issue on Continuous Wavelet Transform in Memory of Jean Morlet, Part I .
[29] D. Braess and W. Hackbusch, On the efficient computation of high-dimensional integrals and the approximation by exponential sums, (2009), p. 39.

[30] W. Wiscombe and J. Evans, Exponential-sum fitting of radiative transmission functions, J. Comput. Phys. 24, 416 (1977).

[31] S. Norvidas, Approximation of bandlimited functions by trigonometric polynomials, Acta Math. Hung. 128, 26 (2010).

[32] For the most general cases, one can set $H_{0}=0$.

[33] M. A. Nielsen and I. L. Chuang, Quantum Computation and Quantum Information: 10th Anniversary Edition (Cambridge University Press, USA, 2011), 10th ed.

[34] A. Kalev and I. Hen, Quantum algorithm for simulating Hamiltonian dynamics with an off-diagonal series expansion, Quantum 5, 426 (2021).

[35] Strictly speaking, they are not standard CNOTs but higherdimensional operations that act like a CNOT on the first two levels.

[36] V. V. Shende, S. S. Bullock, and I. L. Markov, Synthesis of quantum-logic circuits, IEEE Trans. Computer-Aided Design Integrated Circuits Systems 25, 1000 (2006).

[37] J. Dereziński and C. Gérard, Quantum Time-Decaying Hamiltonians. In: Scattering Theory of Classical and Quantum N-Particle Systems (Springer, Berlin, Germany, 1997).

[38] W. Rudin, Principles of Mathematical Analysis (McGrawHill Education, New York City, USA, 1976), 3rd ed. 